\title{
Formation and structure of internal and edge transport barriers
}

\author{
E.J. Synakowski \\ Princeton Plasma Physics Laboratory, Princeton, New Jersey USA
}

\begin{abstract}
The phenomenology of transport barrier formation is reviewed with a focus on physics that may be common to the edge and the core. To this end, the framework of $\mathrm{E} \times \mathrm{B}$ velocity shear reduction of turbulence-induced fluxes, applied to the edge for some time, is studied in light of measurements of core bifurcation dynamics and recent tests of causality. Also, the possible role of the magnetic shear structure in facilitating core barrier formation is examined. Experimental and theoretical challenges for developing predictive capability for reactor-relevant conditions are highlighted by recent observations of spontaneous electric field shear generation far removed from edge effects, and efforts to characterize the plasma edge at and across the L-H transition.
\end{abstract}

Presented at the IAEA Technical Committee Meeting on H Mode Physics

Kloster Seeon, Germany

22-24 September, 1997

This is an expanded version of a paper accepted for publication in Plasma Physics and Controlled Fusion. 


\section{Introduction}

Recent progress in reducing anomalous plasma transport in toroidal devices has been dramatic. The richness of the physics revealed by these developments is a product of vigorous experimental and theoretical efforts worldwide, and opens the possibility of achieving a deeper understanding of nonlinear turbulent plasma phenomena as well as underlying classical processes. While spontaneous bifurcations to enhanced confinement have long been known to occur under the appropriate conditions in the plasma edge, recent experiments underscore that spontaneous reductions in anomalous transport can occur in the core as well. This has led to the remarkable situation in which experimental inferences of small local transport rates in the plasma core are forcing improvements of cross-field neoclassical transport theory [Lin, 1997], a situation generally regarded as unnecessary and even unimaginable just a few years ago.

In addition to spontaneous bifurcations to $\mathrm{H}$ mode confinement that were first observed in the plasma edge [Wagner, 1982], work by [Oren 1982; Taylor, 1989] indicated that similar enhanced confinement states could be induced by externally driving with biased probes changes in the radial electric field. The fact that similar enhanced confinement states could be accessed in these two different ways suggests that the physics might sensibly be divided into two parts, the first part concerning the process of the initial development of the bifurcated state, and the second involving the physics of subsequent edge barrier evolution and sustainment. This division was further suggested for DIII-D H modes [Groebner, 1990; Burrell, 1992] in the form of spectroscopic measurements of impurity poloidal flows and accompanying inferences of spontaneous increases in the $\mathrm{E}_{\mathrm{r}}$ shear that occur before a confinement increase. Interestingly, the history of discovery involving bifurcations to enhanced core confinement has some similarities to that of the edge. Recently observed spontaneous core confinement bifurcations join previously reported regimes with broadly similar confinement characteristics but that are entered with 
external triggers of hydrogen or deuterium pellet injection [e.g. Greenwald, 1984, 1987; Tubbing, 1991]. In light of this, it is reasonable to examine the situation to see if the physics of core transport barrier formation can naturally be separated into two parts as well, and to see therefore if the physics of the edge and the core might be explained in the same framework.

This paper is organized as follows. In Section 2, an overview of core barrier structure and dynamics is given. Analogies are drawn to edge barriers where common themes can be highlighted. Section 3 contains an outline of the basic concepts of $E \times B$ shear suppression as they apply to toroidal plasmas. Section 4 contains a discussion of the local dynamics of spontaneous bifurcations to enhanced edge and core confinement, and reviews experimental data that address the causal role of $E_{r}$ and $E_{r}$ shear effects in sustaining reduced transport in the core and the edge. In Section 5, the possible role of the q profile in facilitating transitions to enhanced confinement and reducing electron thermal transport is discussed. Section 6 concentrates on needs and challenges for theory and experiment in developing a predictive framework for transport bifurcations in a reactor-relevant environment. A summary is presented in Section 7.

\section{Barrier structure, bifurcations, and dynamics}

There are many reviews of the structure and bifurcating properties of edge transport barriers in the literature, including past proceedings of this conference [e.g., Burrell, 1992; Groebner, 1993; Carlstrom, 1996a]. In this section, emphasis is on the structure and dynamics of core bifurcations recently observed in plasmas heated with neutral beams and possibly RF. Comparison to the edge is made where common elements can be highlighted.

Similarities in the radial structure of core and edge barriers are of interest as they may point to common physics involved in the formation and sustainment of both. Such qualitatively similar aspects of core and edge barriers are highlighted in Fig. 1. Shown are edge barrier characteristics in an $\mathrm{H}$ mode plasma from DIII-D, as well as core barrier characteristics of an Enhanced Reverse Shear (ERS) plasma on TFTR. In both, steep pressure gradients are accompanied by large gradients in the radial electric field. In addition, on devices where 
measurements have been made, both core and edge barriers are correlated with local reductions in fluctuation amplitude, or a reduction in fluctuation-induced fluxes. Finally, both core and edge barriers often form in regions that are unique from the standpoint of magnetic topology. In the edge, the foot of the $\mathrm{H}$ mode pedestal is located close to the boundary between open and closed field lines. In the core, barrier formation in some situations may be associated with unique locations in the q profile, e.g. qmin, or integral and near-integral values of $\mathrm{q}$, as is suggested in High $\beta_{\mathrm{p}}$ modes in JT-60U [Koide, 1996], and electron barriers on RTP [DeBaar, 1997].

Any successful theory of core and edge transport barrier formation must successfully describe the nonlinear physics involved in the dynamics of bifurcating systems. On many devices, small differences in the pre-transition "target" plasmas can yield remarkably different plasma evolutions. This is illustrated in Fig. 2. Auxiliary heating waveforms and the electron density peaking factor for two JET optimized shear plasmas [Sips, 1997a] and two TFTR reverse shear plasmas are shown [Synakowski, 1997b]. In the case of the JET plasmas, differences in the heating power are only in the early stages of RF heating, while in the TFTR case, the neutral beam heating power is nearly identical in both cases, even with respect to the details of the neutral beam faults. However, for one plasma in each pair, the electron density peaking undergoes a radically different evolution than the other in that pair, a signature that one has undergone a transition to a state of enhanced core confinement. Prior to the transition, the density and temperature profiles of the two plasmas in each pair are similar.

At least for particles and the ion thermal channel, if the underlying physics of core transport barrier formation can be described in the same framework from one device to the next, it is unlikely that this framework has as a fundamental element the q profile or its shape [Mazzucato, 1996; Synakowski, 1997b]. The similarities in the q profiles just prior to the transition time for a TFTR reverse shear/enhanced reverse shear pair highlight this (Fig. 2e). Another important consideration regarding the role of shear reversal is that there are regimes, such as the High $\beta_{\mathrm{p}} \mathrm{H}$ mode of JT-60U, for which shear reversal is not present, but for which core transport barriers certainly form. This, plus the observation that core transport barrier 
formation with reversed, weakly reversed, or normal shear is generally characterized by a power threshold [e.g. Söldner 1997, Synakowski 1997b, Koide 1996], indicates that shear reversal is neither a sufficient nor a necessary condition for core barrier formation in these plasmas. However, there is evidence that it plays a role as a facilitator in barrier formation in some circumstances. Indications that it may be a key element in the reduction of transport in the electron thermal channel are discussed in Section 5.

Evidence that common physics between devices may be involved in sustaining enhanced confinement after a bifurcation is found by comparing the barrier dynamics. Enhanced core confinement states are often characterized by barrier expansion after the initial transition. Shown in Fig. 3 are the ion temperature profiles for a High $\beta_{\text {p }}$ mode from JT-60U [Koide, 1996], and for a JET optimized shear plasma [Sips, 1997a]. For both, the transport barrier, as defined by the change in the slope of the ion temperature, expands well beyond the radius of the barrier initiation. Such behavior is also reported for the JT-60U reverse shear regime [Fujita 1997b], as well as for DIII-D negative central shear plasmas [Burrell, 1997; Greenfield 1997]. A theoretical model for describing the dynamics of core barrier propagation in the framework of $\mathrm{E} \times \mathrm{B}$ shear turbulence reduction has been outlined by [Lebedev, 1997]. This work suggests that the outward propagation rate is determined primarily by the residual diffusion rates in the core, stopping only when the turbulence decorrelation and stabilization associated with the local gradients at the edge of the high confinement region cannot overcome the radially increasing instability drive.

In the core, improved ion thermal and particle confinement often occur together [Levinton, 1995; Strait 1995; Koide, 1996, Sips, 1997(b,c)]. To this extent, core barrier behavior is analagous to edge behavior. For plasmas with core density diagnostic coverage, like the optimized shear scenario on JET [Sips, 1997b; Söldner 1997], and ERS plasmas of TFTR [Levinton, 1995], rates of increase in the central density are indistinguishable from the integrated particle source rate from neutral beam fueling [Fig. 4]. Upon $\mathrm{H}$ mode formation, additional retention of particles from edge sources is observed. Likewise, intrinsic impurity density buildup that is coincident with the formation of an ion thermal barrier is intrinsic to High $\beta_{p}$ plasmas of 
JT-60U [Koide, 1996]. The prospect of helium ash accumulation with core and edge barriers is therefore of general concern, and emphasizes the need for the development of transport barrier control tools.

\section{Possible roles of $\mathrm{E} \times \mathrm{B}$ shear and magnetic shear reversal in transport barrier formation}

In searching for physics common to both the edge and the core and between devices, much attention has been paid to $\mathrm{E} \times \mathrm{B}$ shear flow effects because of its potential for influencing a wide variety of modes through nonlinear decorrelation of turbulence, and its influence through linear stabilization. While the latter process is generally mode-specific, it can influence the overall stability of a system through coupling between stable and unstable modes [Burrell, 1997]. A review of the theoretical understanding of turbulence decorrelation and stabilization is given in these proceedings [Staebler, 1997]. Below, a brief overview as it pertains to results discussed in this paper is given.

The basic concepts involved in $\mathrm{E} \times \mathrm{B}$ shear decorrelation of turbulence were outlined in the work of [Biglari, 1990], where it was argued that shear in the E $\times$ B plasma flow can lead to decorrelation of fluctuations and reduced transport levels. As originally outlined, it was not immediately obvious how the observed rapid bifurcation character of many transitions to enhanced confinement could be captured by this picture. Later, treatments emerged that selfconsistently evolved effects of $\mathrm{E} \times \mathrm{B}$ shear on turbulence with plasma profile effects [Diamond, 1994; Carreras, 1994; Diamond, 1995], and later with a multimode transport model [Newman, 1997], along with models that evolve plasma profiles through inclusion of nonlinear dependencies of transport coefficients on $\mathrm{E} \times \mathrm{B}$ shear [Hinton, 1993; Staebler, 1997]. These selfconsistent approaches demonstrate that it is possible to reproduce many of the dynamics such as fast bifurcations and barrier propagation observed in the core and edge in this framework.

For any plasma species, evaluation of the terms of the radial force balance equation yields the radial electric field through $E_{r}=\nabla p /(n Z e)+V_{\phi} B_{\theta}-V_{\theta} B_{\phi}$, where $n$ is the density of the species in question, $\mathrm{p}$ is its pressure, $\mathrm{Z}$ is the charge number, e is the electronic charge, $\mathrm{V}_{\theta}$ is its 
poloidal rotation, $\mathrm{V}_{\phi}$ is its toroidal rotation, $\mathrm{B}_{\theta}$ is the poloidal magnetic field, and $\mathrm{B}_{\phi}$ is the toroidal field. In calculations of $\mathrm{E}_{\mathrm{r}}$ reported here and previously elsewhere, $\mathrm{V}_{\theta}$ is calculated from neoclassical considerations. However, recent measurements by [Bell, 1997], discussed below, suggest that the behavior of the poloidal flows in the core can deviate from neoclassical estimates especially at the bifurcation time. While recent developments illustrate that $\mathrm{E}_{\mathrm{r}}$ can be measured directly using the Motional Stark Effect [Zarnstorff, 1997; Rice, 1997], consideration of the force balance equation provides insight into the interplay between $\nabla \mathrm{p}$ and the plasma flow velocity. Since it is the total $E_{r}$ that influences the $E \times B$ velocity and its shear, any one of the three terms in the force balance can potentially play a role in stabilizing turbulence. This sets up the possibility of the existence of various feedback loops by which the influence of $\mathrm{E} \times \mathrm{B}$ shear on turbulence can be reinforced. For example, with centrally deposited neutral beam heating with little net applied torque, the $\nabla \mathrm{p}$ term can dominate the determination of $\mathrm{E}_{\mathrm{r}}$ and its gradient. Therefore, any increases in $\nabla \mathrm{p}$ that occurs with enhanced confinement will lead to larger increases in $\mathrm{E}_{\mathrm{r}}$ and $\mathrm{E}_{\mathrm{r}}$, yielding further stabilization and decorrelation of turbulence. Likewise, plasmas dominated by rotation through external toroidal momentum input can develop a shear in $\mathrm{V}_{\phi}$ that yields a shear in $E_{r}$ that subsequently improves momentum confinement and further increases $V_{\phi}$ shear. Finally, the interplay between $\mathrm{V}_{\theta}$ and the other terms may be potentially important, as is discussed in Section 4.

According to [Hahm, 1995], in a toroidal geometry, it is the gradient in $\mathrm{E}_{\mathrm{r}} / \mathrm{RB}_{\theta}$, and not just $E_{r}$, that characterizes the shearing of turbulence, where $R$ is the major radius. In a tokamak plasma of arbitrary cross sectional shape, a characteristic rate for shearing turbulence can be written as $\gamma_{\mathrm{E} \times \mathrm{B}}=\frac{\Delta \Psi_{0}}{\Delta \phi}\left[\frac{\partial}{\partial \Psi}\left(\frac{\mathrm{E}_{\mathrm{r}}}{\mathrm{RB}_{\theta}}\right)\right]$, where $\Psi$ is the poloidal magnetic flux, $\Delta \mathrm{r}_{0} \equiv \Delta \Psi_{0 / R B_{\theta}}$ is the radial correlation length, and $\Delta \phi$ is the toroidal correlation angle. Assuming isotropy of turbulence in the plane perpendicular to the magnetic field leads to a simpler expression valid on the outer midplane $[H a h m, 1996]$, i.e. $\gamma_{E \times B}=E_{r} / B\left[1 / E_{r}\left(\partial E_{r} / \partial R\right)-1 / B_{\theta}\left(\partial B_{\theta} / \partial R\right)-1 / R\right]$, where $B$ is the total magnetic field magnitude. In lieu of a self-consistent calculation of the growth rates including E $\times \mathrm{B}$ flow effects, advantage is often taken of results from [Waltz, 1994] that indicate 
that complete stabilization takes place in some systems when $\gamma_{\mathrm{E} \times \mathrm{B}} \sim \gamma_{\text {lin }}^{\max }$, where $\gamma_{\text {lin }}^{\max }$ is the fastest linear growth rate of the dominant instability, calculated without including $\mathrm{E} \times \mathrm{B}$ shear effects. Although care must be exercised in applying this figure of merit too rigorously, and the relation between these rates cannot in themselves be used to describe the time scales and dynamics of confinement bifurcations, the ratio $\gamma_{\mathrm{E} \times \mathrm{B}} / \gamma_{\operatorname{lin}}^{\max }$ is used here as a guide to indicate when $\mathrm{E} \times \mathrm{B}$ flow shear effects might be expected to be important in sustaining a transition to enhanced confinement.

In the context of the $\mathrm{E} \times \mathrm{B}$ shear suppression model, reversed magnetic shear in the core can facilitate barrier formation and sustainment in several ways [Synakowski, 1997b]. First, for a given plasma stored energy, a plasma with a hollow core current density profile as compared to one with a peaked current density profile will yield larger Shafranov shift gradients. This leads to both a reduction in expected growth rates and fluxes, and a weakened or inverse dependence of the growth rates on the pressure gradient [Beer, 1997]. Second, compared to plasmas with peaked current density profiles with little toroidal rotation, the larger Shafranov shifts that result from hollow current profiles yield larger values of $\nabla \mathrm{p}$ on the outer midplane and larger values of $\mathrm{E}_{\mathrm{r}}$ and its gradient, leading to increased values of $\gamma_{\mathrm{E} \times \mathrm{B}}$. Third, large values of $1 / \mathrm{B}_{\theta}\left(\partial \mathrm{B}_{\theta} / \partial \mathrm{R}\right)$ that result from a hollow current profile can also lead to an increase in $\gamma_{E \times B}$, depending the shape of $\mathrm{B}_{\theta}$ relative to $\mathrm{E}_{\mathrm{r}}$. Also, for given pressure and velocity profiles, theory predicts a reduction in the calculated microinstability growth rates as a result of reversed magnetic shear itself [e.g., Kodomtsev, 1967; Kessel, 1994; Itoh, 1994b; Beer, 1997].

\section{Local bifurcation phenomenology, fluctuation changes, and tests of causality}

4.1. Fluctuation behavior across the bifurcation in the core and the edge - Whatever the physics of core and edge transport barrier formation and sustainment, most theoretical efforts have concentrated on mechanisms by which microturbulence-induced fluxes are suppressed. One requirement of a successful framework is that it should account for the fact that turbulence amplitudes and/or turbulence-induced fluxes are suppressed even as sources of free energy, such 
as temperature and velocity gradients, continue to increase. The framework of $\mathrm{E} \times \mathrm{B}$ shear suppression outlined above has this character.

In devices where core fluctuation measurements are possible, density fluctuation amplitude reduction is found at the onset and after confinement bifurcations. These observations are suggestive of the longer history of reductions in turbulence levels and turbulence-induced fluxes at the edge of the L-H transition, indicating that confinement improvement is achieved by some means of microinstability suppression in both cases. Shown in Fig. 5(a,b) are the central electron density, measured with interferometry, and the electron density fluctuation amplitude, measured with reflectometry [Mazzucato, 1996] just inside the radius the eventually becomes the boundary of the barrier, for a TFTR ERS plasma. The high power period of injection started at $2.5 \mathrm{~s}$. The density fluctuations have a bursting character as a function of time, suggesting a competition between instability drive and a suppression mechanism. These core fluctuation levels fall abruptly at the transition time, where the transition time is inferred from the rate of rise of the central density. The elements of a self-sustaining bifurcation through positive feedback is suggested, as $\gamma_{\mathrm{E} \times \mathrm{B}} / \gamma_{\text {lin }}^{\max }$ increases after the transition. The increasing value of this ratio after the transition is facilitated by the stabilization due to the large Shafranov shift. Remarkably, using the calculated neoclassical value of $V_{\theta}, \quad \gamma_{E \times B} \sim \gamma_{\text {lin }}^{\max }$ at the transition time. This may be deceptively suggestive: recent studies indicate that the shearing rate estimated at these times are likely underestimated owing to measured large excursions in the poloidal rotation velocity near this time, as is discussed below. In addition, variations by a factor of two in the ratio of the two rates at the time of turbulence suppression was observed in nonlinear calculations [Waltz, 1994], and similar variations were found in threshold scaling experiments in which the toroidal field was varied [Levinton, 1996; Synakowski, 1997b].

An important prediction of the picture of $\mathrm{E} \times \mathrm{B}$ shear stabilization of turbulence is the fact that the sign of $E_{r}$ and $\gamma_{E \times B}$ is not important in reducing turbulence. As such, unidirectional beam injection in the direction with the plasma current should yield a positive contribution to $\mathrm{E}_{\mathrm{r}}$ from the $V_{\phi} B_{\theta}$ term in the radial force balance. A net positive $E_{r}$ is thus inferred for most Negative 
Central Shear (NCS) plasmas on DIII-D, unlike the negative electric field that is inferred in the plasma edge during $\mathrm{H}$ mode operation and in the core of TFTR ERS plasmas. Like TFTR, however, core density fluctuation amplitudes are found to disappear nearly completely in the high power phase of injection [Burrell, 1997; Rettig, 1997]. In fact, the fluctuations that may persist generally do so on the high field side of the plasma. Owing to the behavior of the scale length of the poloidal magnetic field, the high field is the region in which the $\mathrm{E} \times \mathrm{B}$ shearing rate is the lowest. This unexpected anti-ballooning character of the density fluctuation amplitudes is therefore consistent with the presence of $\mathrm{E} \times \mathrm{B}$ velocity shear reducing the turbulence.

Similar to these recent observations in the core, reductions in fluctuation-induced fluxes also occur in the edge at the L-H transition [Tynan, 1994; Moyer, 1995]. Shown in Fig. 5(d,e) are measurements from DIII-D [Moyer, 1995] of the edge electron density across an L-H transition, measured with a lithium beam diagnostic, and fluctuation amplitude at the edge, measured with reflectometry. The improvement in confinement is well correlated with turbulent fluctuation amplitude reduction. Like the core of NCS and ERS plasmas, turbulence-induced flux reductions through $\mathrm{E} \times \mathrm{B}$ shear stabilization provides a self-consistent framework for sustained confinement improvement in this edge in the face of steepened temperature and density gradients. Although the $\mathrm{E}_{\mathrm{r}}$ generation begins through the spontaneous generation of a poloidal flow shear (Fig. 6; discussed below), it is ultimately dominated by an increased pressure gradient after the transition.

4.2. Spontaneous $E_{r}$ changes in the core and the edge - One of the hallmarks of transitions to enhanced confinement in the edge is the spontaneous development of increased electric field strength and its gradient. Consequently, a major challenge continues to be identification of the underlying physics responsible for this bifurcation. In addition to the previously mentioned computational efforts indicating that bifurcations are possible in the context of $\mathrm{E} \times \mathrm{B}$ shear stabilization if pressure gradient and rotation effects are included self-consistently, models of electric field bifurcations driven by plasma particle losses [Itoh and Itoh, 1988, 1989a, 1989b, 1990, 1996], viscosity-driven radial currents [Shaing, 1989] and Stringer spin-up of poloidal 
flow [Hassam, 1991] have all been put forward. More recent efforts have concentrated on the role of turbulence-driven Reynolds stress-induced flow generation [Diamond and Kim, 1991; Diamond 1994], and initial experimental work has begun to assess the viability of this picture in tokamaks and stellarators [Balbin 1997].

It has been reported that the change in $\mathrm{E}_{\mathrm{r}}$ in edge $\mathrm{H}$ mode transitions on some tokamaks first becomes apparent in the plasma poloidal rotation [Groebner, 1990; Burrell, 1992]. For the impurity ions, this appears as a localized poloidal rotation excursion in the electron diamagnetic drift direction just inside the separatrix (Fig. 6). On DIII-D, this change in poloidal rotation represents a spontaneous increase in $\mathrm{E}_{\mathrm{r}}$ shear that precedes measurable changes in the other background plasma profiles, pointing to a threshold character of the confinement bifurcation and indicating that the change in $\mathrm{E}_{\mathrm{r}}$ shear is a causal element of the transport bifurcation. The change in confinement can be identified by the rapid change in the electron density near the radius of changes in $\mathrm{E}_{\mathrm{r}}$ ' that occurs after the initial stages of poloidal rotation change. After the change in confinement is initiated, the pressure gradient increases and eventually assumes the role of the poloidal rotation in dominating the determination of $E_{r}$ in the impurity force balance equation. This indicates that the physics of the barrier formation might be viewed distinctly from the physics of barrier sustainment. Precursors in the impurity poloidal rotation have also been found in the W7-AS stellarator [Wagner 1994], suggesting that the trigger observed on some tokamaks might be operative more generally. The early domination of poloidal rotation in edge $\mathrm{E}_{\mathrm{r}}$ changes in the transition to the H mode has also been reported on JFT-2M [Ida et al, 1990, 1992], although from this work it is not clear whether the poloidal rotation change precedes the $\mathrm{H}$ mode transition. It should be noted, however, that $\mathrm{E}_{\mathrm{r}}$ in $\mathrm{H}$ modes on JET is dominated by the pressure gradient contribution at all times, and shows no indication of a poloidal rotation precursor [Hawkes 1995] within the measurement resolution. In addition, although increases in spatially averaged measurements of $\mathrm{E}_{\mathrm{r}}$ prior to an $\mathrm{H}$ mode transition are inferred from neutral particle analysis on ASDEX-U [Herrman, 1995, 1997; Zohm, 1996], these are slow compared to the local $E_{\mathrm{r}}$ excursions seen on DIII-D. 
Recent measurements of impurity poloidal rotation made in the core of ERS plasmas on TFTR indicate that the picture of a two stage $\mathrm{H}$ mode formation process can be extended to the area of core barrier formation in some circumstances. The electric field evolution in this case generally consists first of a poloidal rotation precursor, followed by $\nabla \mathrm{p}$ dominance of $\mathrm{E}_{\mathrm{r}}[\mathrm{Bell}$, 1997; Synakowski, 1997c] (Fig. 6). The precursor is located close to the minimum in the q profile. The change in $\mathrm{V}_{\theta}$ clearly precedes any change in local confinement characteristics. At the peak of the poloidal flow excursion, an increase in the local confinement, as represented in the figure by the local carbon pressure, takes place. As $V_{\theta}$ subsequently relaxes, the pressure gradient drive begins to dominate the determination of $\mathrm{E}_{\mathrm{r}}$. This phenomenology is remarkably similar to that reported for DIII-D H mode barrier formation. Recent direct measurements of the local electric field are consistent with the spectroscopic determination that the $\mathrm{V}_{\theta}$ excursion is the dominant component of the electric field early in time [Bell, 1997], as is found in the $\mathrm{H}$ mode. It is important to note that at least one example of an ERS transition without an observed excursion of the carbon $\mathrm{V}_{\theta}$ just prior to the change in confinement exists, suggesting that the excursion may be a facilitating but not a necessary feature of the bifurcation.

Pursuing similarities between the core and edge transitions further, the spontaneous $\mathrm{E}_{\mathrm{r}}$ shear development is exceptionally well localized in both cases. In the core of TFTR, it challenges the $3 \mathrm{~cm}$ chordal spacing of the viewing chords. The need to identify the origin of this precursor in $\mathrm{E}_{\mathrm{r}}$ shear evolution, already a subject of active consideration regarding the $\mathrm{H}$ mode for many years, is now reemphasized with these observations in the core. The fact that $V_{\theta}$ returns to pre-excursion values after the confinement bifurcation suggests that ERS transitions are not caused by a sustained neoclassical poloidal viscosity bifurcation like that proposed for $\mathrm{H}$ mode transitions by [Shaing, 1989]. However, it is consistent with a fluctuation-driven flow generation which is terminated when the fluctuations are suppressed [Diamond, 1991; Diamond, 1994].

\subsection{Tests of causality with externally modified electric fields - A challenge for demonstrating} causality for the role of $\mathrm{E}_{\mathrm{r}}$ or $\mathrm{E} \times \mathrm{B}$ shear in generating or sustaining enhanced confinement 
resides in the fact that confinement improvements caused by any mechanism whatsoever are characterized by increases in pressure gradients or velocity shear. As such, increased electric field shear is usually an effect, but may not be a cause, of confinement improvement. Experiments using externally applied changes to the electric field have the potential of breaking this relation between electric field gradients that normally accompany steep pressure or velocity shears. Recently, such work has been extended to include studies performed with edge probes on TEXTOR [Jachmich, 1997; Weynants, 1997], and modifications of the core radial electric field through variations in the neutral beam torque on TFTR [Synakowski, 1997a,b].

An important aspect of the TEXTOR work is that evidence indicates that electric field shear, and not $\mathrm{E}_{\mathrm{r}}$ effects per se, are responsible for modifying confinement. A discussion and illustration of the time histories of the ramp of the applied bias, the measured electric field and electric field gradient response, electron density gradient response, and particle confinement are given in these proceedings [Weynants, 1997] and elsewhere [Jachmich, 1997], and an overview is given here. In these experiments, cause and effect were separated by imposing positive electric fields with electrode biasing that oppose the $\mathrm{E}_{\mathrm{r}}$ normally established by the pressure gradient. A mushroom-shaped electrode was placed $5 \mathrm{~cm}$ inside the ALT-II limiter, and electric field profiles were measured with a nine-tip rake probe. Early in the ramp, changes in $\mathrm{E}_{\mathrm{r}}{ }^{\prime}$ are correlated spatially and temporally with changes in the density gradient and improvements in particle confinement prior to any bifurcation in the electrode current. The density gradient changes least where the electric field is a maximum, and $E_{r}^{\prime}$ is nearly zero (Fig. 7). This spatial alignment between $E_{r}^{\prime}$ and $\nabla n_{e}$ indicates that it is $E_{r}^{\prime}$, and not $E_{r}$, that is responsible for the enhanced confinement state. After about $1 \mathrm{~s}$ in the bias ramp, a bifurcation in the electrode current occurs simultaneously with a bifurcation in the plasma potential, leading to a state of $\mathrm{H}$ mode-like confinement. Interestingly, the incremental increase in $\nabla n_{e}$ is proportional to the magnitude of the increase in $E_{r}^{\prime}$, for both signs of $E_{r}^{\prime}$, prior to the bifurcation. However, this symmetry is broken after the bifurcation. The origin of the difference in the $\mathrm{H}$ mode state is not known, and future tests with negatively biased probes are planned to examine this issue. Finally, oscillations 
in $E_{r}$ ', caused by a ringing of the biased probe power supply after the current bifurcation, provide a useful test of causality. These slightly lead the oscillations in $\nabla \mathrm{n}_{\mathrm{e}}$, again pointing to the causal role of $E_{r}^{\prime}$ in reducing anomalous transport.

The role of $\mathrm{E} \times \mathrm{B}$ shear in sustaining enhanced confinement was examined in the core of ERS plasmas on TFTR through forced variations in $E_{r}$ and the $E \times B$ shearing rate [Synakowski, 1997a,b] created through applied variations of the neutral beam torque at constant heating power. Steady-state ERS plasmas were generated by reducing neutral beam power by nearly a factor of two below that required to initiate the transition. The $14 \mathrm{MW}$ thus used after the forward transition was equivalent to the total power available for injection either parallel or anti-parallel to the plasma current, permitting significant variations in the toroidal velocity $\mathrm{V}_{\phi}$. These variations yielded corresponding changes in $E_{r}$ and its shear with nearly constant heating power and similar heating source profiles. This kept quantities central to other proposed means of turbulence stabilization, such as the gradient in the Shafranov shift $\Delta^{\prime}$ and the peaking of the density profile, constant during the ERS phase, and allowed electric field effects to be separated from other stabilizing influences.

The plasma pressure and global energy confinement time remained nearly constant throughout the period in which $\mathrm{V}_{\phi}$ variations were applied so long as the discharges remained in the ERS regime. However, reducing the electric field and its shear with co-rotation reproducibly triggered a back-transition (Fig. 8). The sum of the $\nabla \mathrm{p}$ and $\mathrm{V}_{\theta} \mathrm{B}_{\phi}$ terms of the force balance equation was aligned with the $\mathrm{V}_{\phi} \mathrm{B}_{\theta}$ term such that reductions in $\mathrm{E} \times \mathrm{B}$ shear were achieved with co-rotation. For different degrees of co-rotation, back-transitions occurred at different times but for similar values of $\gamma_{\mathrm{E} \times \mathrm{B}}$. For any particular plasma, the causal role of $\mathrm{E} \times \mathrm{B}$ shear is emphasized by the fact that reductions in $\gamma_{\mathrm{E} \times \mathrm{B}}$ precede back-transitions, while all other plasma quantities, including the plasma pressure and $\Delta^{\prime}$, are constant or nearly constant in time (Fig 9). The fact that core confinement is unchanged above a threshold value of $\gamma_{\mathrm{E} \times \mathrm{B}}$ indicates that at least some class of the microturbulence may be completely suppressed when $\gamma_{\mathrm{E} \times \mathrm{B}}$ is above a critical value, consistent with the nonlinear calculations of [Waltz, 1994]. Increases in core local fluctuation 
levels, measured from reflectometry, are correlated with increases in local transport coefficients in plasmas with back-transitions. In similar plasmas, the measured $\mathrm{V}_{\theta}$ remains constant during the ERS period, indicating that it is indeed the $\mathrm{V}_{\phi}$ change that is forcing the change in $\mathrm{E}_{\mathrm{r}}$. These results are analagous to those obtained in magnetic braking experiments in VH modes on DIII-D, where the introduction of a small magnetic perturbation induced a torque on the plasma. The forced reduction in toroidal rotation shear and the $\mathrm{E} \times \mathrm{B}$ shearing rate was accompanied by a loss of enhanced confinement as well [LaHaye, 1995].

4.4. Limit cycles in the edge and the core - Limit cycle behavior has been observed with both core and edge transport barriers, again illustrating that some of the dynamics of core and edge barriers have common elements. In both cases reported here, the role of the electric field has been suggested as being central to the observed behavior. At low $\mathrm{B}_{\mathrm{T}}$, the TFTR ERS power threshold is low enough to permit a core barrier to be formed with the application of significant net neutral beam torque. In such a plasma whose high power phase was dominated by coinjection power, multiple transitions into and out of enhanced confinement were observed, as noted by the central electron density peaking factor (Fig. 10). This dithering behavior is consistent with a competition between the sum of the $\nabla p$ and the $V_{\theta} B_{\phi}$ terms of the carbon force balance equation, which yield a negative contribution to $\gamma_{\mathrm{E} \times \mathrm{B}}$ near the barrier location, and the $\mathrm{V}_{\phi} \mathrm{B}_{\theta}$ term, which yields a positive contribution. In light of the back transition work discussed above, this interpretation is reinforced by noting that it is only after the total power is reduced by reducing the momentum input to nearly zero that a sustained transition to the ERS regime is realized. Dithering into and out of the $\mathrm{H}$ mode state at the plasma edge on ASDEX-U has been examined by [Zohm, 1995]. In this work, the sequential transport bifurcations are explained in terms of a limit cycle inherent in the electric field development and the pressure gradient. The picture outlined only requires that the electric field be a multivalued function of plasma parameters, such as that proposed by [Itoh, 1991], to capture the essential character of the limit cycle. The elements common to the interpretation of the results in the edge and the core are that 
the competing conditions for turbulence suppression reinforcement (increases in the edge power flow in the $\mathrm{H}$ mode; and increases in the pressure gradient in the ERS plasma) are out of phase with elements that help determine the minimum stabilization requirements (rapid edge density buildup in the $\mathrm{H}$ mode; rapid toroidal velocity shear buildup in the ERS plasma).

\section{The $q$ profile and confinement improvement}

Understanding the physics of the behavior of the electron channel presents a significant challenge for experiment and theory. Initial reports of the formation of core transport barriers indicated that the origin of most of the confinement improvement resided in changes in the ion thermal channel, but that the electron thermal channel was comparatively stubborn in its response [Levinton, 1995; Lao, 1996; Forest, 1996]. However, studies on JT-60U [Fujita, 1997a; Fig. 11] indicate that electron thermal confinement can be improved with central heating, and that the improvement might be facilitated with magnetic shear reversal [Koide 1997a]. Similar results were recently reported for DIII-D plasmas [Burrell, 1997]. On JT-60U, a substantial pedestal in the electron temperature profile is observed with strongly reversed shear, and power balanced analysis indicates that there is a decrease in the electron thermal conductivity, $\chi_{\mathrm{e}}$ [Fujita, 1997a]. While a systematic study pointing to the causal role of the q profile in reducing $\chi_{\mathrm{e}}$ has yet to be performed, the results indicate that reduction of transport in the electron channel is possible.

Further observations of reduced $\chi_{\mathrm{e}}$ in the core of reverse shear discharges were reported for Tore Supra plasmas [Litaudon, 1996a,b]. Transitions to enhanced confinement are noted by spontaneous increases in the central electron temperature [Fig. 12]. With these increases, there is inferred from power balance a marked drop in $\chi_{\mathrm{e}}$. The electron thermal barrier forms only when the magnetic shear is below a critical value of approximately zero, indicating that shear reversal may be a critical component of the electron thermal channel suppression. Using these correlations, a model based on the assumption that $\chi_{\mathrm{e}}$ is an explicit function of magnetic shear has been used to explore the dynamics of Tore Supra discharges. The model self-consistently evolves the bootstrap current and the thermal transport, and reveals that core electron 
temperature oscillations observed during lower hybrid heating can be reproduced under these assumptions.

Perhaps connected to the suggestion that near-integer values of q may be related to barrier dynamics in High $\beta_{\mathrm{p}}$ plasmas [Koide, 1996], observations made on the RTP tokamak suggest that barrier formation is closely tied to integer or nearly integer values of the minimum in the $q$ profile. With reversed magnetic shear formed by off-axis ECH heating, nonlinear confinement responses in the core are found when qmin passes through 3 (Fig. 13) [de Baar, 1997]. Above this value, enhanced core confinement is not observed. While the different values of $\mathrm{q}_{\min }$ are generated by small variations in the radii of $\mathrm{ECH}$ deposition, it is important to note that these variations are a small fraction $(<10 \%)$ of the width of the ECH heating location and of the width

of the electron thermal transport barrier itself. More recent studies have confirmed the highly nonlinear nature of the barrier formation physics through both current profile scans and toroidal field ramps.

\section{Challenges for experiment and theory}

While considerable progress has been made in characterizing bifurcations and outlining possible frameworks for the physics involved, many challenges must be met before this picture matures into a useful predictive framework. Understanding the origin of spontaneous electric field shear increases in the edge and core prior to a confinement change presents a significant challenge. While there is evidence that radial currents can play a leading role in the dynamics of transport bifurcations in some circumstances, spontaneous $\mathrm{E}_{\mathrm{r}}$ shear increases deep in the core suggests the possibility of the existence of fluctuation-driven self-regulated poloidal flows. In fact, both the edge and the core have elements that are broadly considered to be important for such flow generation through the Reynolds stress [Diamond and Kim, 1991; Diamond, 1994]. Two such elements include fluctuation inhomogeneity (the edge is known to have large radial gradients in fluctuation amplitudes, while shear reversal is thought to create a radial asymmetry 
in instability drive) and anisotropy in the turbulent eddies (which may exist if the eddy size rivals the background plasma profile scale lengths).

Local influences such as wall neutrals and radiation may be of profound importance to $\mathrm{H}$ mode transition physics, yet they are not included in any bifurcation theory in a self-consistent fashion. A demonstration of how they might affect local dynamics was recently performed [Moyer, 1997] in $\mathrm{H}$ modes for which a significant fraction of the input power was radiated by Marfes outside the X-point. Bifurcations unfolded in a manner similar to more familiar "fast" transitions, i.e. precursor poloidal rotation changes and subsequent bifurcations in confinement were observed, but with time scales 50 - 100 times longer than seen in previous studies. It has been proposed that the radiative losses inhibit the supporting role of the pressure gradient in accelerating the bifurcation process, and thus increase the transition time.

Significant differences in density and potential fluctuation behavior were inferred in the work of [Tynan, 1994], as well as that of [Moyer, 1995]. In [Moyer, 1995], measurements of density and potential fluctuations in the shear suppression zone were made in ohmic and ohmic $\mathrm{H}$ mode conditions [Fig. 14]. A general reduction in density and potential fluctuations is found shortly after the bifurcation. Eventually, however, the fluctuation amplitudes return to near or above their pre-transition values as the pressure gradient increases, even though enhanced confinement is maintained. Still, the fluctuation-driven particle fluxes are reduced across the $\mathrm{E}_{\mathrm{r}}$ well, even where $\mathrm{E}_{\mathrm{r}}^{\prime} \sim 0$. The cause of this is the fact that the two fluctuating fields are out of phase where the shear is zero, resulting in little net flux. Most L-H transition models, however, have only a single fluctuating field, and would therefore never successfully capture the results of this experiment. A recent exception to this is the work of [Isichenko, 1995].

An interesting feature is common to recent efforts to characterize the edge at the $\mathrm{L}-\mathrm{H}$ transition. On every tokamak that has examined the issue, the edge temperature just prior to the transition is restricted to a narrow range [Hubbard, 1997; Carlstrom, 1996b; Groebner, 1996; Righi, 1997; Zohm, 1996]. There is no consensus at present as to how this is to be viewed in terms of the requirements for spontaneous electric field generation. Although this narrow range 
of edge temperatures might point to ion orbit loss physics as being important for the generation of $E_{r}$, relatively poor correlation between the power threshold and the ion collisionality $v^{*}{ }_{i}$ was found due to the wide range of densities examined in some of these studies [Carlstrom, 1996b; Groebner, 1996], consistent with the studies of [Ida, 1992]. These results indicate that, in their simplest forms, theories for the generation of the edge electric field that depend on a critical value of $v^{*}$, such as the ion orbit loss model [Shaing, 1989], do not contain the general features necessary to capture the essential physics of spontaneous electric field generation that takes place in these plasmas. However, local effects, such as neutrals and magnetic field ripple, may play a role in the wide variation of $v^{*}$. For example, work by [Fukuda, 1997] on JT-60U suggests that the role of neutrals may in part explain the existence of $\mathrm{H}$ mode transitions with $v^{*} \mathrm{i}$ well below unity.

\section{Summary}

Emphasis has been placed on identifying basic properties of transport barriers common to the edge and the core. In both, barriers are identified with steep gradients in the electric field. Fluctuation amplitude reduction measured in the core finds a counterpart in the edge through measurements of reductions in fluctuation amplitudes at the $H$ mode transition, and accompanying reductions in fluxes. A theme common to many transitions in the core and the edge is that they have a threshold character, highlighting the fact that a successful treatment of the edge and the core dynamics is a fundamentally nonlinear one. While both edge and core transitions are often associated with unique locations related to the magnetic field structure, shear reversal is neither a necessary or sufficient condition for transitions to enhanced core confinement, at least in the particle and ion thermal channels.

Similarities in the local dynamics of edge and the core barrier formation are also seen in the poloidal rotation behavior of impurities and the accompanying electric field shear. Recent measurements on TFTR point to a spontaneous excursion in the core poloidal rotation velocity that precedes the onset of ERS confinement. The analog for this at the edge has been reported 
previously on DIII-D. No explanation is given for how the electric field shear might be spontaneously generated, and understanding its origin remains a significant challenge for the plasma physics community. As the generation of $\mathrm{E}_{\mathrm{r}}$ shear in the TFTR core takes place far from edge effects, the possibility of self-generated plasma flows is suggested.

The picture of $\mathrm{E} \times \mathrm{B}$ shear stabilization as a means for sustaining enhanced confinement has been tested with external variations of the electric field made in the edge and in the core. On TEXTOR, biased probe measurements indicate that it is the electric field gradient, and not $\mathrm{E}_{\mathrm{r}}$, that is correlated with local confinement improvements. On TFTR, variations in the applied input torque at constant heating power allow the $\mathrm{E} \times \mathrm{B}$ shearing rate to be reduced while keeping the pressure gradient constant. The resulting losses of enhanced confinement at low shearing rates point to the causal role of electric field effects in sustaining enhanced confinement in these plasmas.

There is evidence that reverse shear facilitates barrier formation in some circumstances, and may be necessary for electron thermal barrier formation. This is highlighted by results from JT-60U, and more recently from DIII-D. In addition, correlations between electron thermal transport and magnetic shear have been reported in Tore Supra plasmas. Electron thermal barriers were also formed in RTP with reverse shear operation, and their existence is correlated with $\mathrm{q}_{\min }$ falling below a critical value.

Work in characterizing the conditions required for $\mathrm{H}$ mode transitions, and the dynamics of bifurcations under different conditions, highlight issues that a successful predictive picture must address. A successful theory of edge bifurcations with meaningful predictive power must include the role of the physics of edge effects and open field lines. Finally, nonlinear physics tools are required to develop predictive capability for both the core and the edge. These will need to evolve electric field shear and turbulence characteristics of multiple fields self-consistently within a toroidal geometry, with heat and particle sources from experiment. Accordingly, significant experimental challenges must be met to measure the elements that are central to the theory. 
Acknowledgments - The author would like to express his appreciation for the valuable discussions and generous contributions from many throughout the community. Those contributing include S. Batha, M. Beer, R.E. Bell, J. Boedo, K. Burrell, C.E. Bush, T. Carlstrom, B. Chapman, M. de Baar, P.H. Diamond, P.C. Efthimion, A. Fujisawa, R. Groebner, T.S. Hahm, A. Hubbard, St. Jachmich, Y. Koide, B. LeBlanc, F. Levinton, X. Litaudon, E. Mazzucato, D. Mikkelsen, R. Moyer, D. Newman, C. Rettig, G. Rewoldt, G. Schmidt, S.D. Scott, A.C.C. Sips, F. Söldner, G.M. Staebler, D.M. Thomas, M.C. Zarnstorff, and H. Zohm. This work was supported under DoE Contract No. DE-AC02-76-CH-03073.

\author{
References \\ Balbin R et al 1997 Proc.24th EPS Conf. on Controlled Fusion and Plasma Physics Berchtesgaden \\ Beer M et al 1997 Phys. Plasmas 41792 \\ Bell R E 1997b Plasma Phys. Control. Fusion (this conference). \\ Biglari H et al 1990 Phys. Fluids B 21 \\ Burrell K H et al 1992 Plasma Phys. Control. Fusion 341859 \\ Burrell K H 1997 Proc.16th IAEA Fusion Energy Conference (Montreal) (Vienna, International Atomic Energy \\ Agency, in press) \\ Burrell K H 1997 Phys. Plasmas 41499 (1997a). \\ Burrell K H 1997 Plasma Phys. Control. Fusion (this conference) \\ Carlstrom T N 1996a Plasma Phys. Control. Fusion 381149. \\ Carlstrom T N and Groebner R J 1996b Phys. Plasmas 31867. \\ Carreras B et al, 1994 Phys. Plasmas 14014 \\ de Baar M R et al 1997 Phys. Rev. Lett. 784573 \\ Diamond P H and Kim Y B 1991 Phys. Fluids B 31626 \\ Diamond P H et al 1994 Phys. Rev. Lett. 722565 \\ Diamond P H et al 1997 Phys. Rev. Lett. 781472 \\ Forest C B et al 1996 Phys. Rev. Lett. 773141 \\ Fukuda T et al 1997 Proc.16th IAEA Fusion Energy Conference (Montreal) (Vienna, International Atomic Energy \\ Agency, in press) \\ Fujita T 1997a et al Phys. Rev. Lett 78 \\ Fujita T et al 1997b Proc.24th EPS Conf. on Controlled Fusion and Plasma Physics Berchtesgaden \\ Greenwald M et al 1984 Phys. Rev. Lett. 534352 \\ Greenwald M et al 1987 Plasma Phys. Contr. Nucl. Fusion Research (Kyoto) (Vienna: International Atomic \\ Energy Agency) vol 1 p 139 \\ Groebner R J 1993 Phys. Fluids B 52343 \\ Groebner R J et al 1990 Phys. Rev. Lett. 643015 \\ Groebner R J et al Proc.16th IAEA Fusion Energy Conference (Montreal) (Vienna, International Atomic Energy \\ Agency, in press) \\ Hahm T S and Burrell K H 1995 Phys. Plasmas 21648 \\ Hahm T S and Burrell K H 1996 private communication \\ Hassam A B et al 1991 Phys. Rev. Lett. 66309
}


Hawkes N 1995 Plasma Phys. Control Fusion 1261

Herrman W et al 1995 Phys. Rev. Lett. 754401

Herrman W et al 1997 Plasma Phys. Control. Fusion (this conference)

Hinton F L Phys. Fluids B 19913696

Hinton F L and Staebler G M 1993 Phys. Fluids B 51281

Holzhauer E et al Proc.24th EPS Conf. on Controlled Fusion and Plasma Physics Berchtesgaden

Hubbard A et al Proc.16th IAEA Fusion Energy Conference (Montreal) (Vienna, International Atomic Energy Agency, in press)

Ida K et al 1992 Phys. Fluids B 42552

Isichenko M B and P H Diamond 1995 Phys Plasmas 22007

Itoh S-I and K. Itoh 1988 Phys. Rev. Lett. 602276.

Itoh S-I et al 1989a Plasma Phys. Control. Nuclear Fusion Research (International Atomic Energy Agency Vienna 1989) vol. 2 p 23

Itoh S -I and K. Itoh 1989b Nucl. Fusion 291031

Itoh S -I and K Itoh 1990 J. Phys. Soc. Japan 593815

Itoh S -I et al 1991 Phys. Rev. Lett. 672485

Itoh K and S -I Itoh et al 1994 Plasm. Phys. Control. Fusion 36279

Itoh K and S -I Itoh 1996 Plasma Phys. Control. Fusion 381

Jachmich St. et al 1997 "Experimental investigations on the effect of E×B flow shear in improved confinement", ERM report \#107, submitted to Plasm. Phys. Control. Fusion

Kessel C et al 1994 Phys. Rev. Lett. 721212

Kishimoto Y et al 1996 Phys. Plasmas 31289.

Kodomtsev B B and Pogutse O P 1966 Zh. Eksp. Teor. Fiz. 511734 [Sov. Phys. JETP 241172 (1967)]

Koide Y et al, in Burrell et al 1994 Plasma Phys. Control. Fusion 1221

Koide Y et al 1996 Plasm. Phys. Control. Fusion 381011

Koide Y and the JT-60 Team 1997a Phys. Plasmas 41623

Koide Y 1997b private communication

LaHaye R et al 1995 Nucl. Fusion 35988

Lao L L et al 1996 Phys. Plasmas 31951

Lebedev V B and Diamond P H 1997 Phys. Plasmas 41087

LeBlanc B P et al 1995 Phys. Plasmas 2741

Levinton F et al 1995 Phys. Rev. Lett. 754421

Levinton F et al 1996 Proc.16th IAEA Fusion Energy Conference (Montreal) (Vienna, International Atomic Energy Agency, in press)

Lin Z et al 1997 Phys. Plasmas 41707.

Litaudon X et al 1996a Plasma Phys. Contr. Fusion 381603

Litaudon X et al 1996b Proc.16th IAEA Fusion Energy Conference (Montreal) (Vienna, International Atomic

Energy Agency, in press)

Mattor N and P H Diamond 1988 Phys. Fluids 311180

Mazzucato E et al 1996 Phys. Rev. Lett. 773145

Messiaen A M et al 1994 Nucl. Fusion 34825

Moyer R A et al 1995 Phys. Plasmas 22397

Moyer R.A. et al 1997 Plasma Phys. Control. Fusion (this conference)

Newman D N et al 1997 submitted to Phys. Plasmas

Oren L et al 1982 J. Nucl. Mater. 111-112 34

Rettig C et al 1997 submitted to Phys. Plasmas

Rice B et al Proc.24th EPS Conf. on Controlled Fusion and Plasma Physics Berchtesgaden

Righi E et al 1997a Plasma Phys. Control. Fusion (this conference)

Righi E et al 1997b Proc.16th IAEA Fusion Energy Conference (Montreal) (Vienna, International Atomic Energy

Agency, in press) 
Schilling G et al 1997 Proc.24th EPS Conf. on Controlled Fusion and Plasma Physics Berchtesgaden

Sips A C C 1997a private communication

Sips A C C et al 1997b submitted to Plasma Phys. Contr. Fusion Research

Sips A C C et al 1997c Plasma Phys. Control. Fusion (this conference)

Söldner F 1997 Proc.24th EPS Conf. on Controlled Fusion and Plasma Physics Berchtesgaden

Staebler G M et al 1994 Phys. Plasmas 1909

Staebler G M Plasma Phys. Control. Fusion (this conference)

Shaing K and E C Crume 1989 Phys. Rev. Lett. 632369

Strait E J et al 1995 Phys. Rev. Lett 754421

Synakowski E J et al 1997a Phys. Rev. Lett. 782972

Synakowski E J et al 1997b Phys. Plasmas 41736

Synakowski E J et al 1997c Proc.24th EPS Conf. on Controlled Fusion and Plasma Physics Berchtesgaden

Taylor R et al 1989 Phys. Rev. Lett. 632365

Tubbing B J D et al 1991 Nucl. Fusion 31839

Wagner F et al 1982 Phys. Rev. Lett. 491408

Wagner F et al 1994 Plasma Phys. Control. Fusion 36 A61

Waltz R E et al 1994 Phys. Plasmas 12229 (1994).

Weynants R et al 1997 Plasma Phys. Control. Fusion (this conference)

Zarnstorff M C et al 1997 Phys. Plasmas 41097

Zohm H et al 1995 Plasma Phys. Control. Fusion 37437

Zohm H et al 1996 Proc.16th IAEA Fusion Energy Conference (Montreal) (Vienna, International Atomic Energy

Agency, in press) 

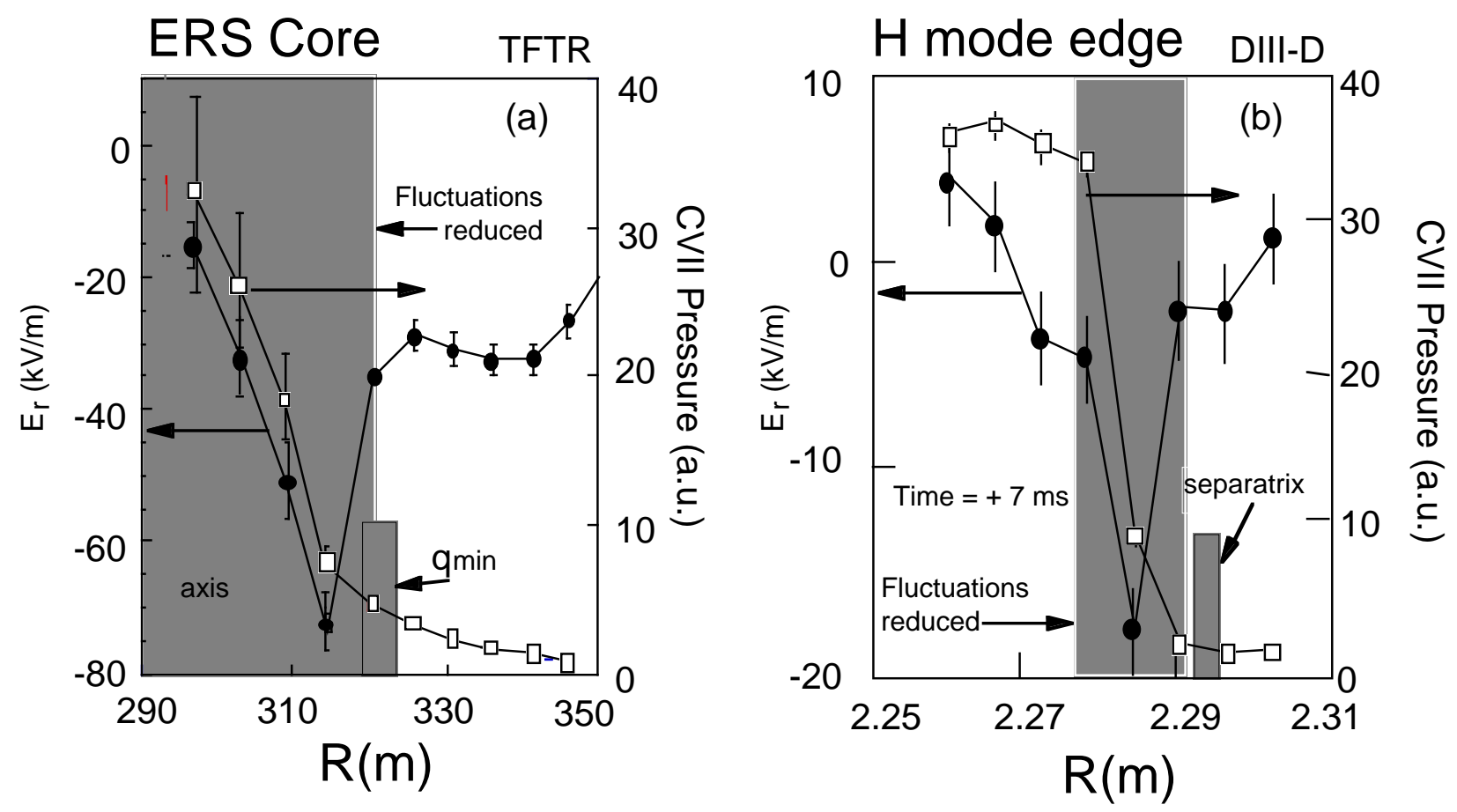

Figure 1. Radial electric field and carbon pressure for (a) a TFTR ERS plasma, and (b) a DIII-D H mode [Groebner, 1993]. The shaded regions represent regions of suppression of turbulence or turbulence-induced fluxes. 

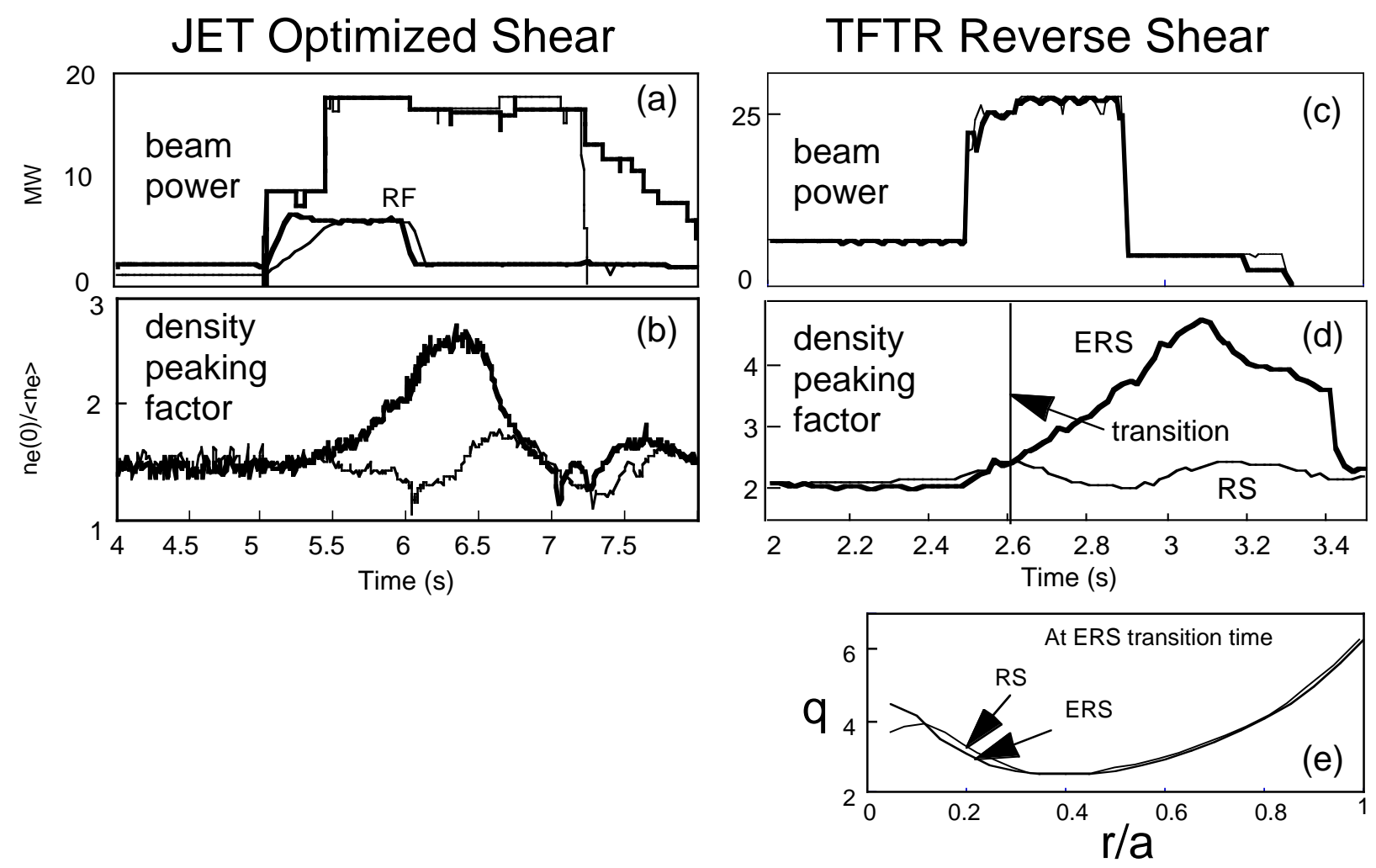

Figure 2. Auxiliary heating waveforms and electron density peaking factors for two pairs of reverse shear plasmas, illustrating the similar nonlinear bifurcating character of entry into these core enhanced confinement regimes. Shown are waveforms for $(a, b)$ optimized shear JET plasmas [Sips, 1997a], and (c,d) TFTR reverse shear plasmas [Synakowski, 1997b]. (e). The q profiles for the two TFTR reverse shear plasmas at the time of the transition of the Enhanced Reverse Shear (ERS) mode, indicating that there is little measurable difference between the two. 

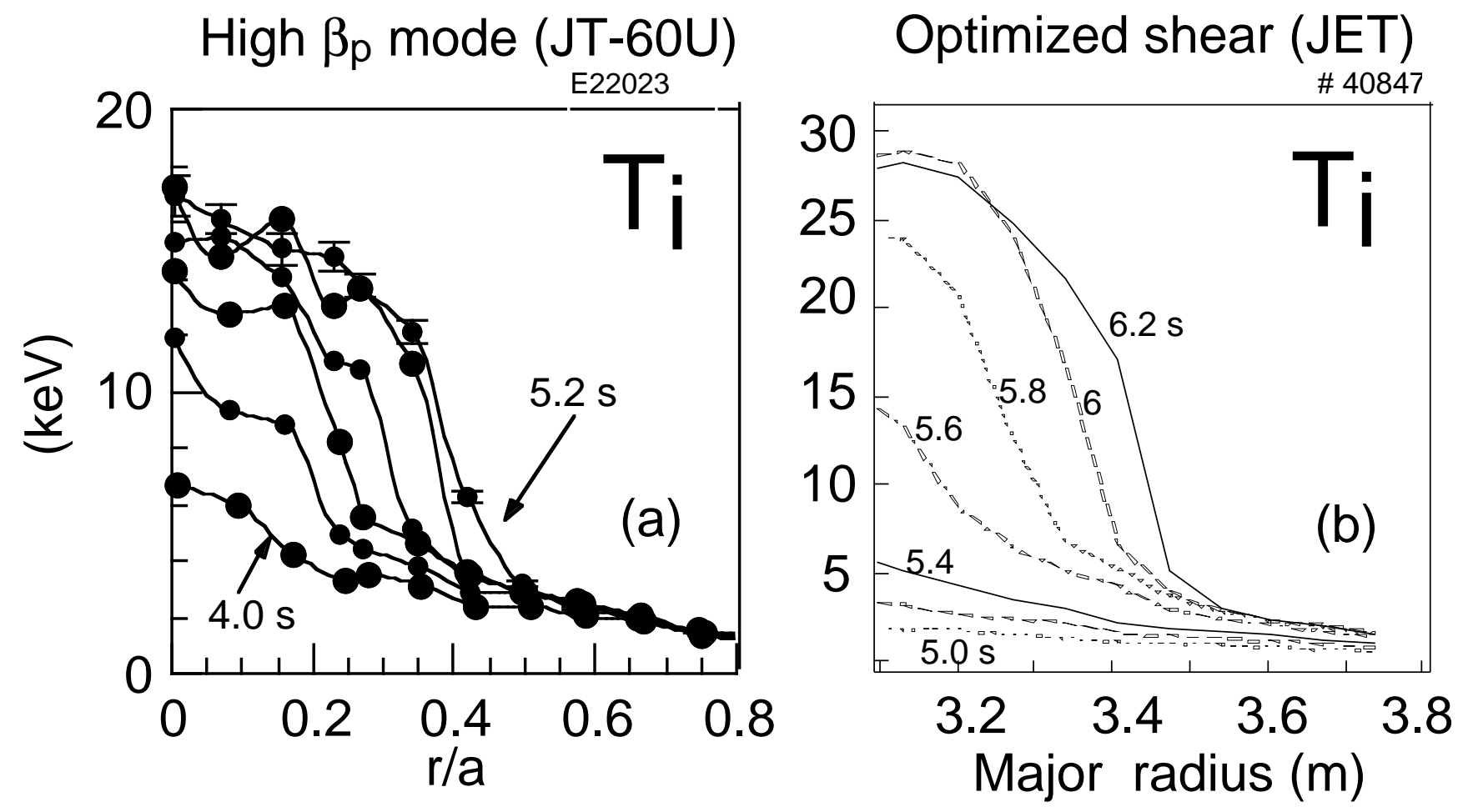

Figure 3. Ion temperature profiles after transitions to enhanced confinement for (a) a High $\beta_{\mathrm{p}}$ mode plasma with a monotonic q profile from JT-60U [Koide, 1996], and (b) a JET Optimized Shear plasma with weak or negative magnetic shear [Sips, 1997b]. For both regimes, the barrier is initiated deep in the core and propagates outward with time. 


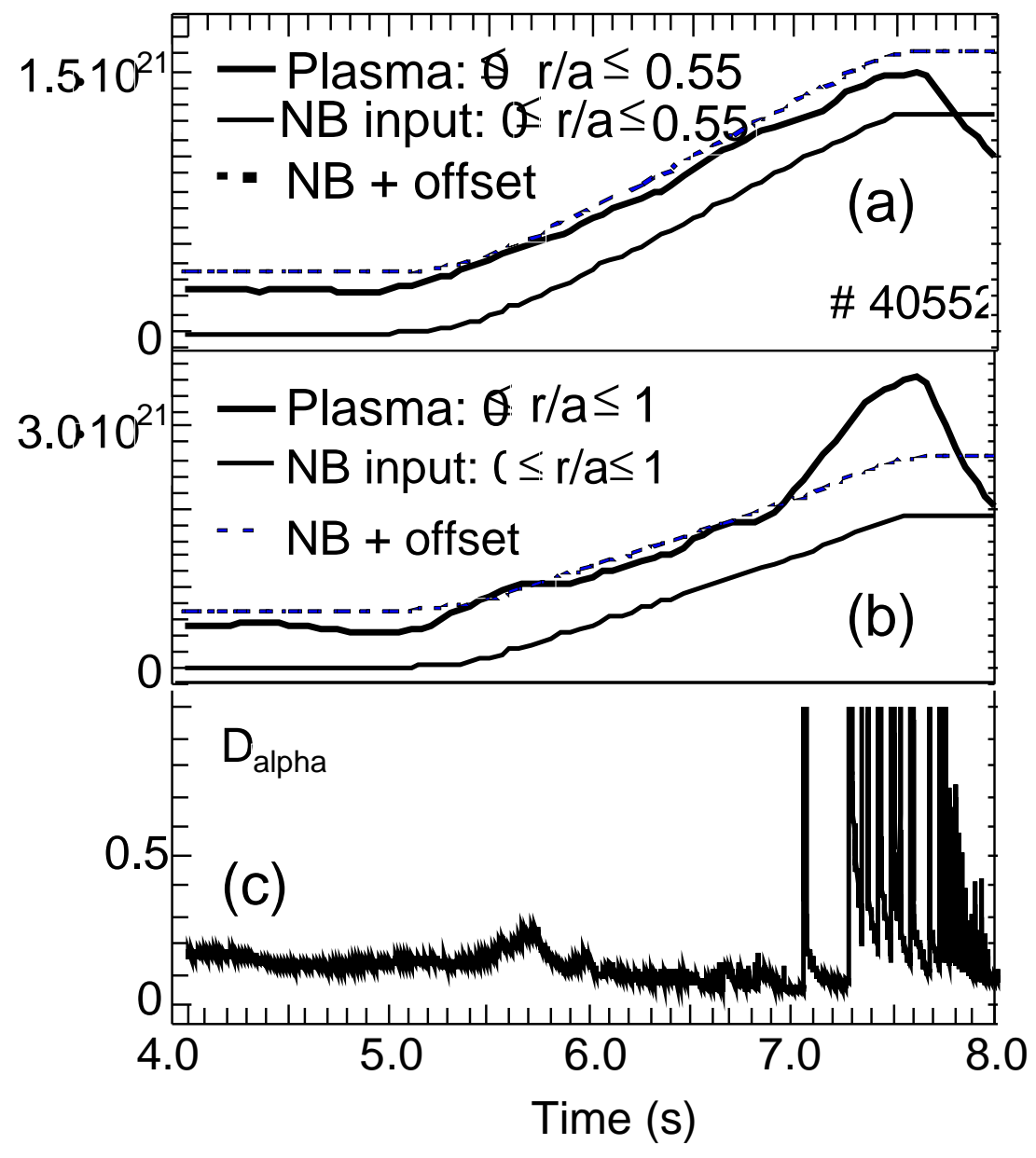

Figure 4. The measured total particle number, and time- and volume-integrated beam particle fueling rates, for a JET Optimized Shear plasma. (a). integrated from $0<\mathrm{r} / \mathrm{a}<1$. (b) integrated from $0<\mathrm{r} / \mathrm{a}<0.55$. The measured rate of increase of the core density is nearly the calculated integrated particle fueling rate from neutral beams. (c). $\mathrm{D}_{\alpha}$ emission from the divertor, showing the onset of an $\mathrm{H}$ mode that leads to an increase in the total particle inventory due to improved retention of particles from edge sources. From [Sips, 1997b]. 

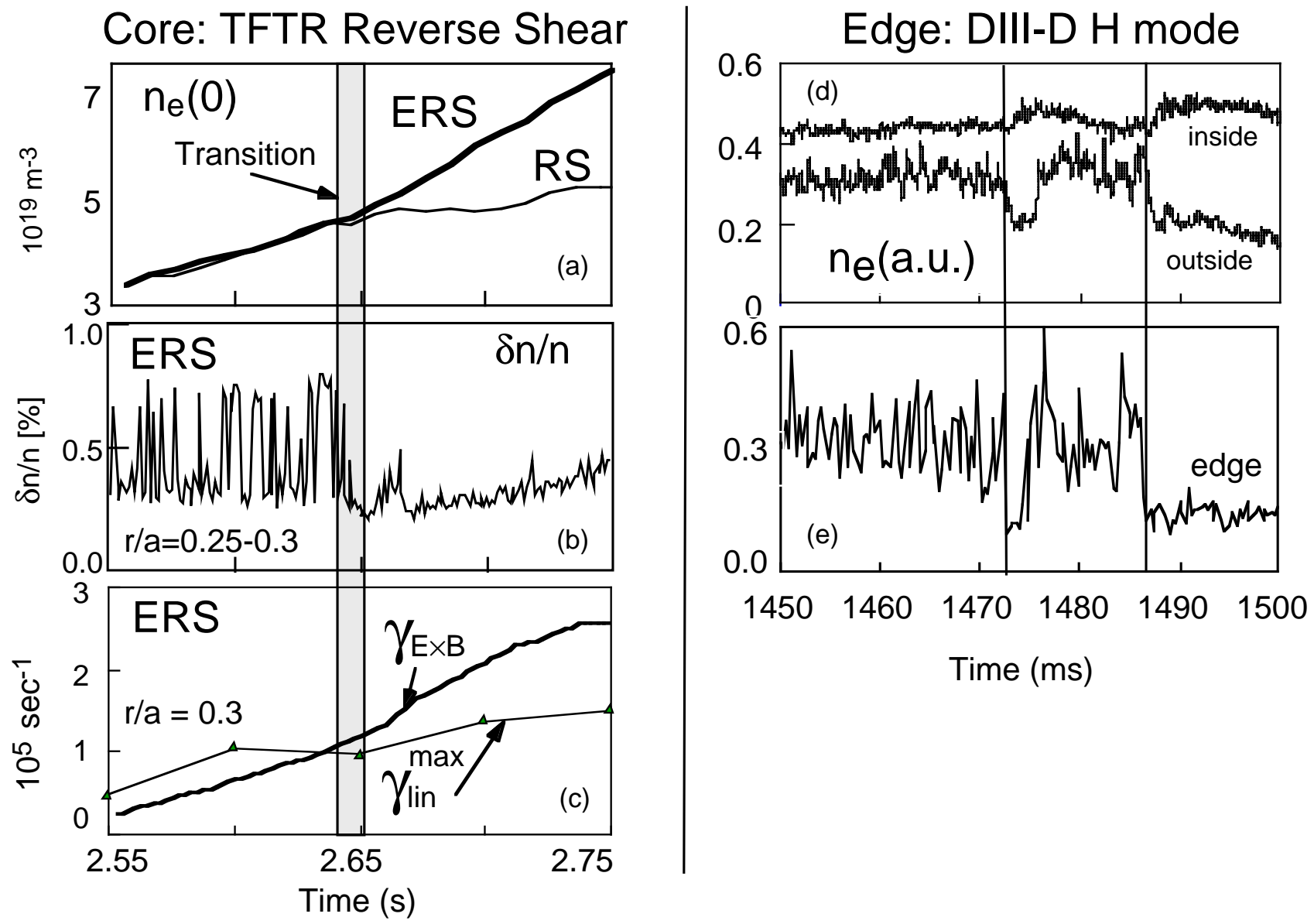

Fig. 5. (a) The central electron density for an ERS plasma and an RS plasma with similar neutral beam heating powers. The transition time is manifest in the change in the rate of rise of the $n_{e}(0)$, marked by the shaded vertical bar. (b). Electron density fluctuations, measured with reflectometry, near the boundary of the region of good confinement. The measurements have an estimated spatial resolution of $1-2 \mathrm{~cm}$, and sample a wavenumber range of $0.5<\mathrm{k}<2.0 \mathrm{~cm}^{-1}$. (c). $\gamma_{\mathrm{E} \times \mathrm{B}}$ and $\gamma_{\text {lin }}^{\max }$ for the ERS plasma, using the calculated impurity poloidal rotation velocity. (d). The local electron density, as measured by a lithium beam diagnostic, for two locations near the separatrix. The vertical lines represent the transition times as defined by this measurement. (e). The fluctuating scattered power, measured by reflectometry, at the edge for the $\mathrm{H}$ mode. The ERS data are from [Synakowski, 1997b], and the DIII-D H mode data are from [Moyer, 1995]. 

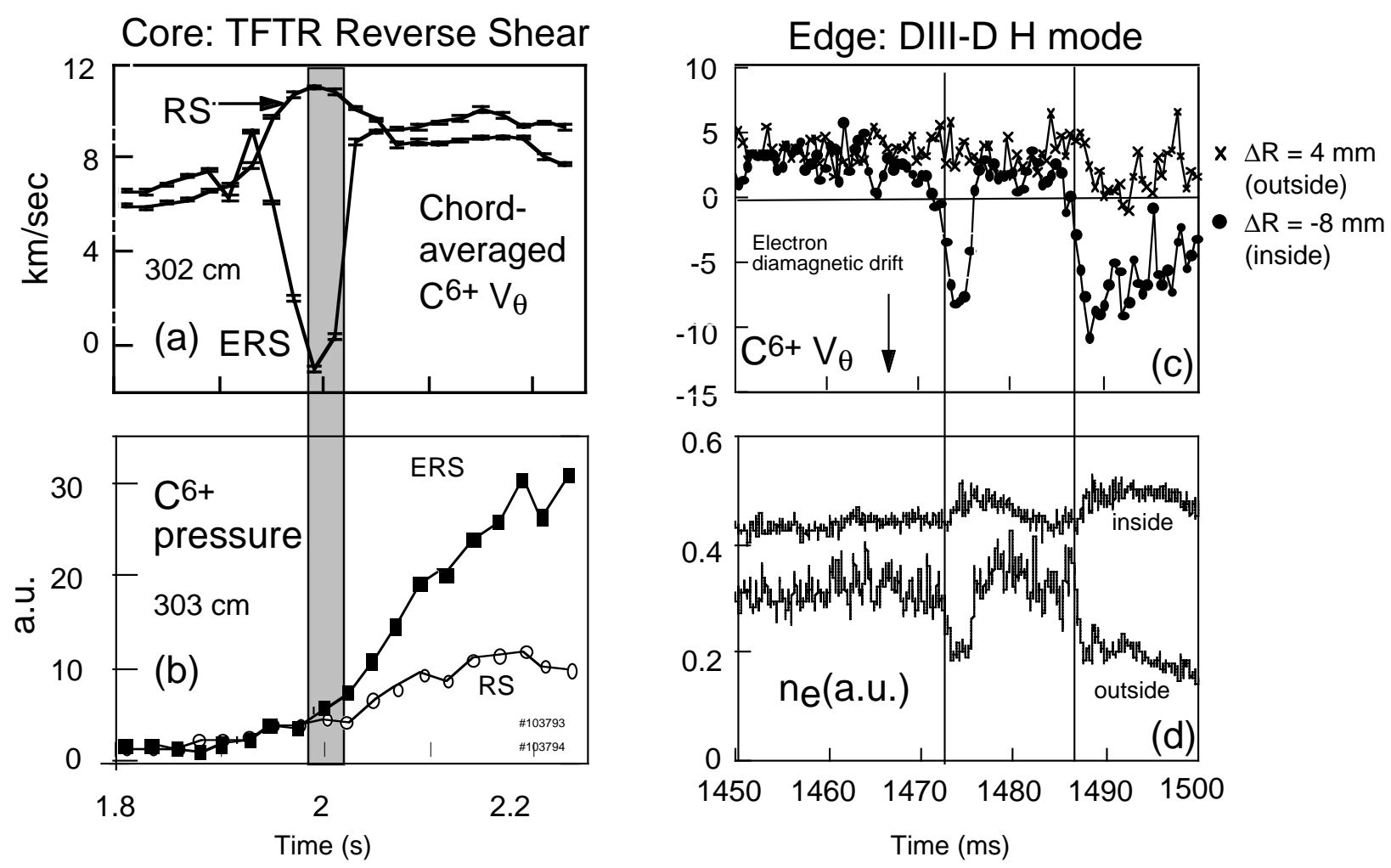

Figure 6. Characteristics of local parameters of the L-H transition on DIII-D and ERS transition on TFTR. (a) Carbon poloidal rotation velocity [Bell, 1997] for two TFTR reverse shear plasmas. The velocity excursion occurs only for the ERS plasma, is located just inside the minimum in the q profile, and occupies a region no wider than a few $\mathrm{cm}$. The excursion begins before the transition to enhanced confinement, as illustrated by (b), the change in the local carbon pressure. (c). Carbon poloidal rotation for the DIII-D H mode [Burrell, 1994; Moyer, 1995]. The change in rotation is just inside the separatrix. Like the ERS plasma, the change begins before the bifurcation in confinement. (d) The local electron density, as measured by a lithium beam diagnostic, for two locations near the separatrix. Later in time, the electric field is dominated by the pressure gradient for both cases. 

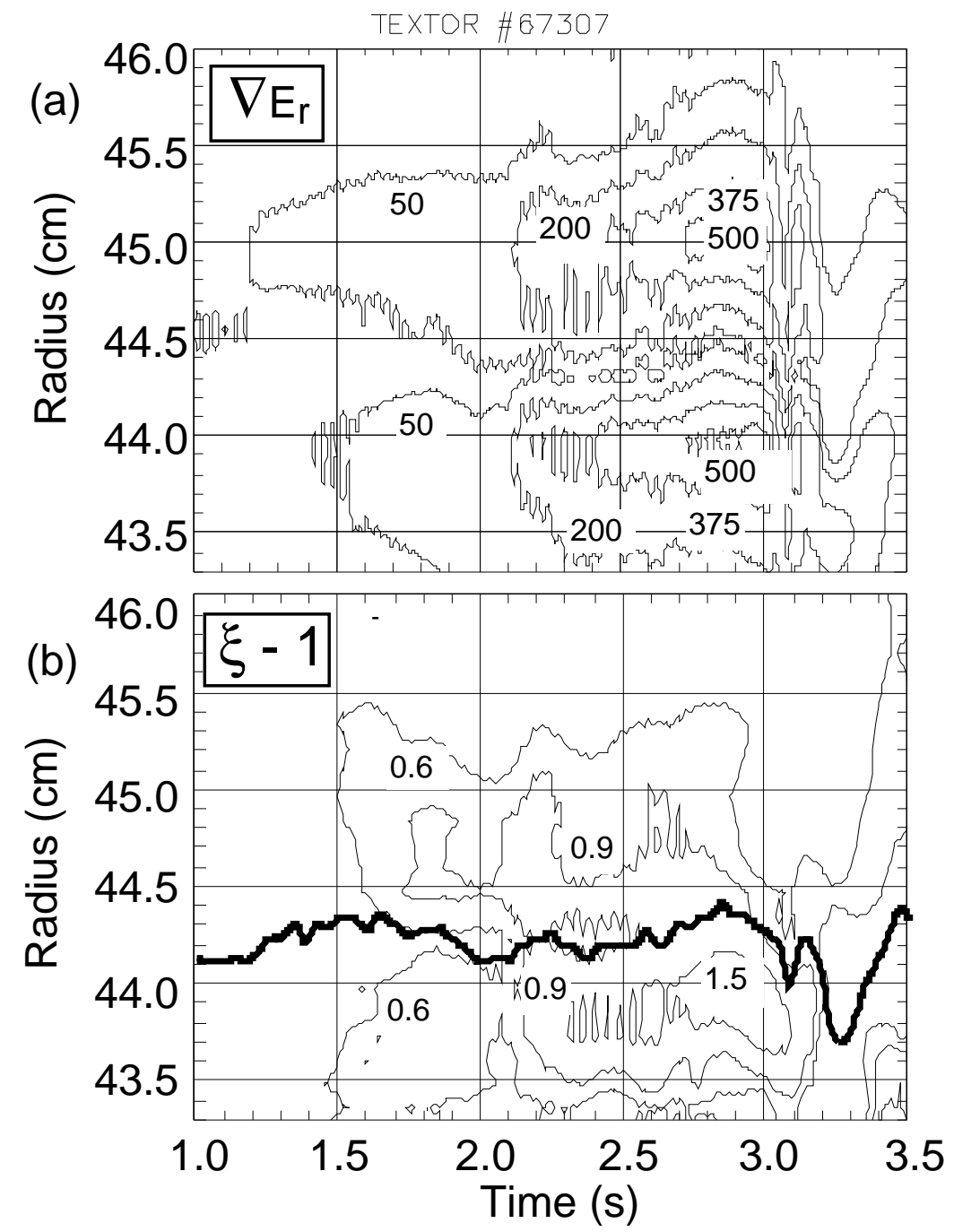

Figure 7. Contour plots of (a) the relative change $\xi-1 \equiv \nabla n_{\mathrm{e}} / \nabla \mathrm{n}_{\mathrm{e}}(0)$ of the density gradient and (b) the absolute value of the electric field gradient $\nabla E_{\mathrm{r}}$ in $\left[\mathrm{V} / \mathrm{cm}^{2}\right]$ (the outer maxima corresponds to the negative field gradient side). The thick sold line indicates the position of the electric field maximum. The ramp begins at $1.0 \mathrm{~s}$; a spatial correlation between changes in $\nabla E_{r}$ and changes in $\nabla n_{\mathrm{e}}$ is observed, even before a bifurcation to $\mathrm{H}$ mode-like confinement that takes place near $2.15 \mathrm{~s}$. The minimum change in $\nabla \mathrm{n}_{\mathrm{e}}$ occurs at the top of the applied $\mathrm{E}_{\mathrm{r}}$ hill, where $\nabla \mathrm{E}_{\mathrm{r}}$ $=0$. From [Jachmich, 1997; Weynants, 1997] 


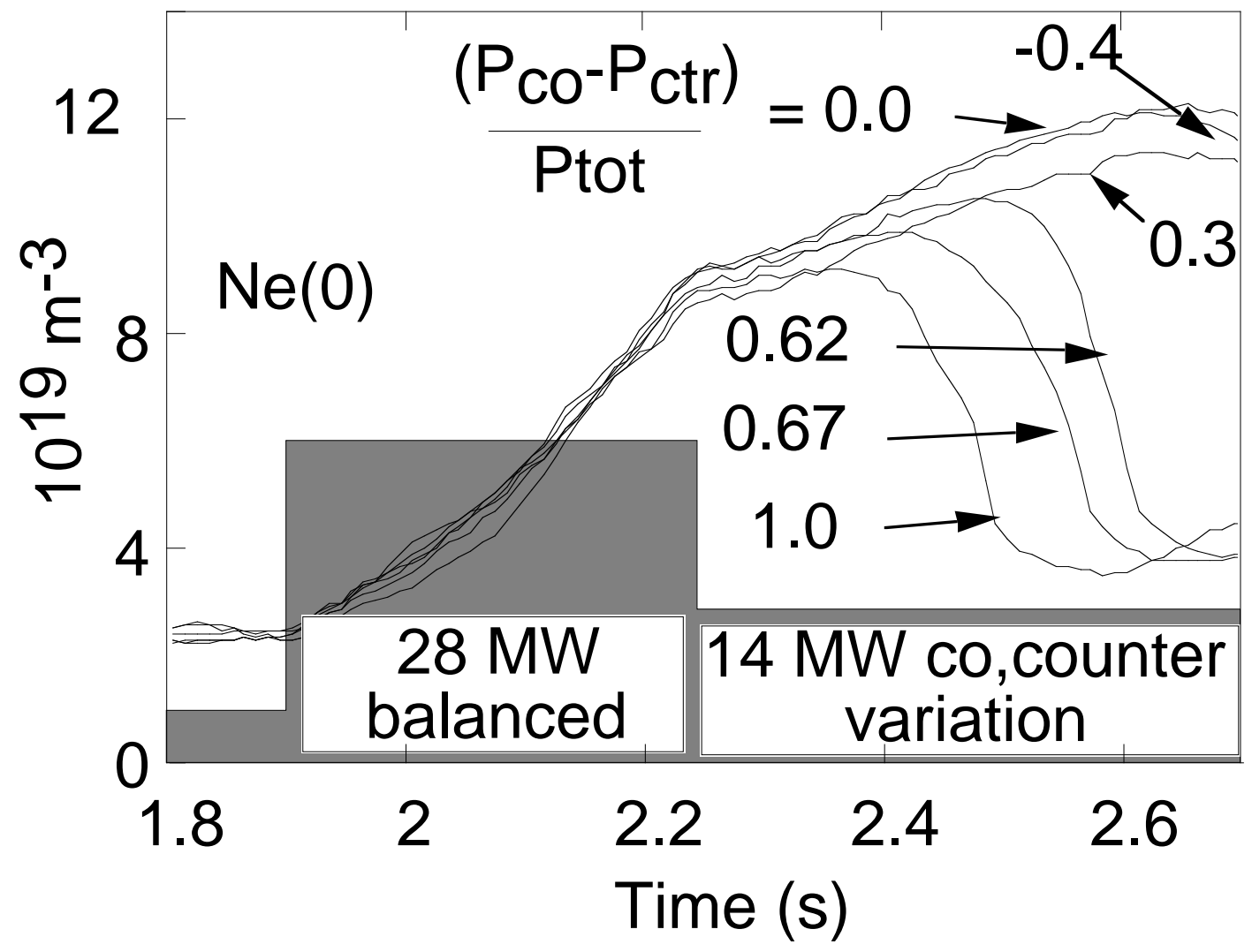

Figure 8. The central electron density time evolution in the TFTR ERS barrier relaxation (back transition) study. Curves are labeled according to the difference on co- vs. counter-injected power, $\mathrm{P}_{\mathrm{co}}-\mathrm{P}_{\mathrm{ctr}}$, divided by the total injected power $\mathrm{P}_{\text {tot }}$, in the $14 \mathrm{MW}$ period. The shaded region indicates schematically the neutral beam heating waveform. From [Synakowski, 1997a]. 


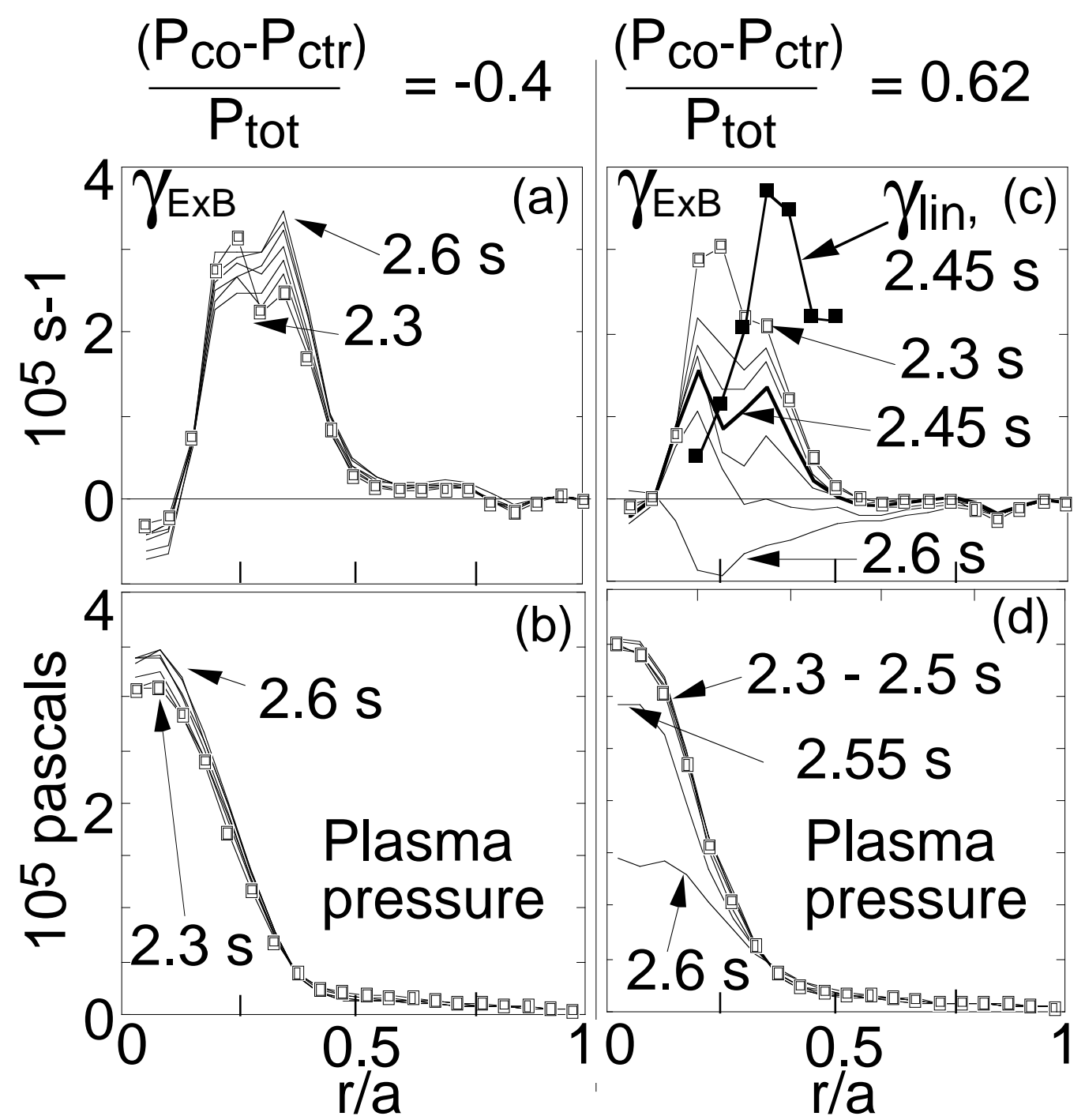

Figure 9. Profiles of shearing rate and total plasma pressure for a counter-dominated plasma $\left[\left(\mathrm{P}_{\mathrm{co}}-\mathrm{P}_{\mathrm{ctr}}\right) / \mathrm{P}_{\text {tot }}=-0.4\right](\mathrm{a}, \mathrm{b})$, and one of the co-dominated plasmas $\left[\left(\mathrm{P}_{\mathrm{co}}-\mathrm{P}_{\mathrm{ctr}}\right) / \mathrm{P}_{\text {tot }}=0.62\right](\mathrm{c}, \mathrm{d})$. In the co-dominated case, the plasma pressure is unchanged until $\gamma_{\mathrm{E} \times \mathrm{B}}$ falls well below $\gamma_{\text {lin }}^{\max }$. From [Synakowski, 1997a]. 


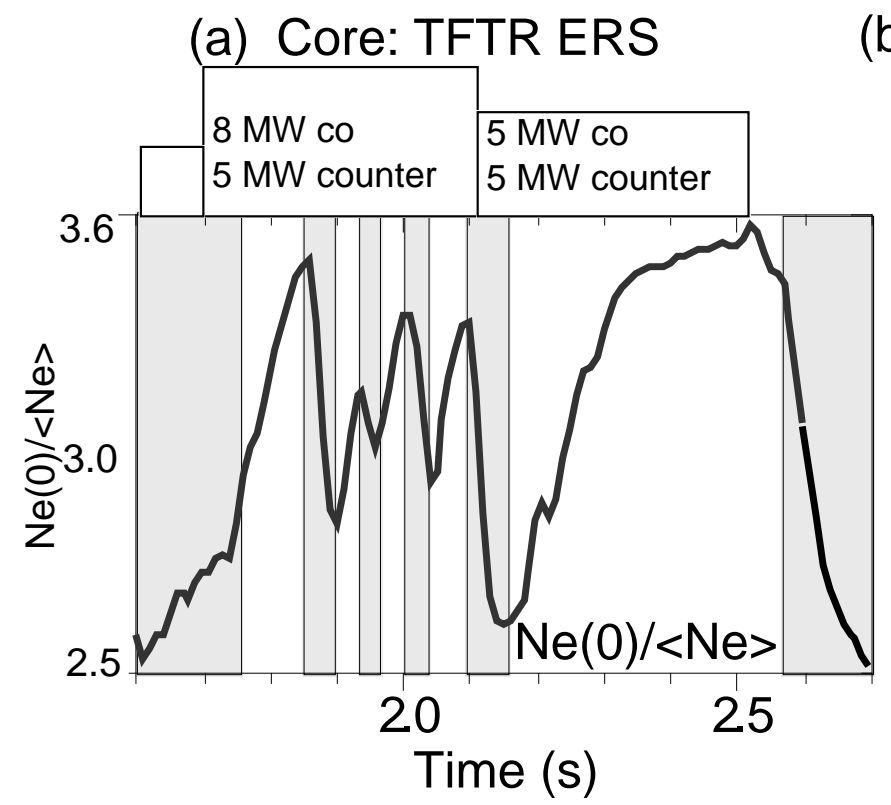

(b) Edge: ASDEX-U H mode

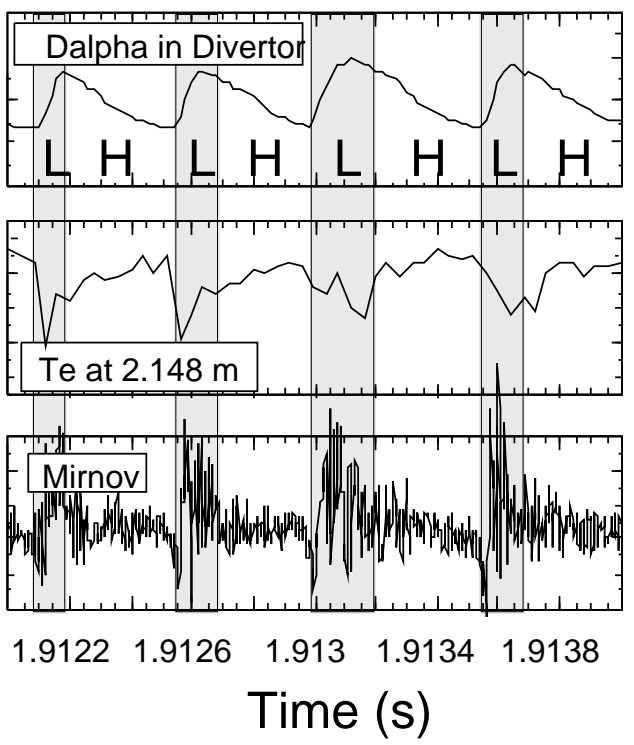

Figure 10. Examples of dithering between low and enhanced confinement states in the core and the edge. (a). The density peaking factor $n_{e}(0) /</ n_{e}>$ for a TFTR ERS plasma. The data are consistent with a limit cycle governed by negative contributions to the shearing rate from the $\nabla \mathrm{p}$ and $\mathrm{V}_{\theta} \mathrm{B}_{\phi}$ terms of the carbon force balance equation, and positive contributions from the $\mathrm{V}_{\phi} \mathrm{B}_{\theta}$ term. (b). $\mathrm{D}_{\alpha}, \mathrm{T}_{\mathrm{e}}$, and Mirnov coil data for an ASDEX-U plasma. The data are consistent with $\mathrm{H}$ mode transition and back transition conditions being governed by $E_{r}$, provided that $E_{r}$ is a multivalued function of plasma parameters [Zohm, 1995]. 

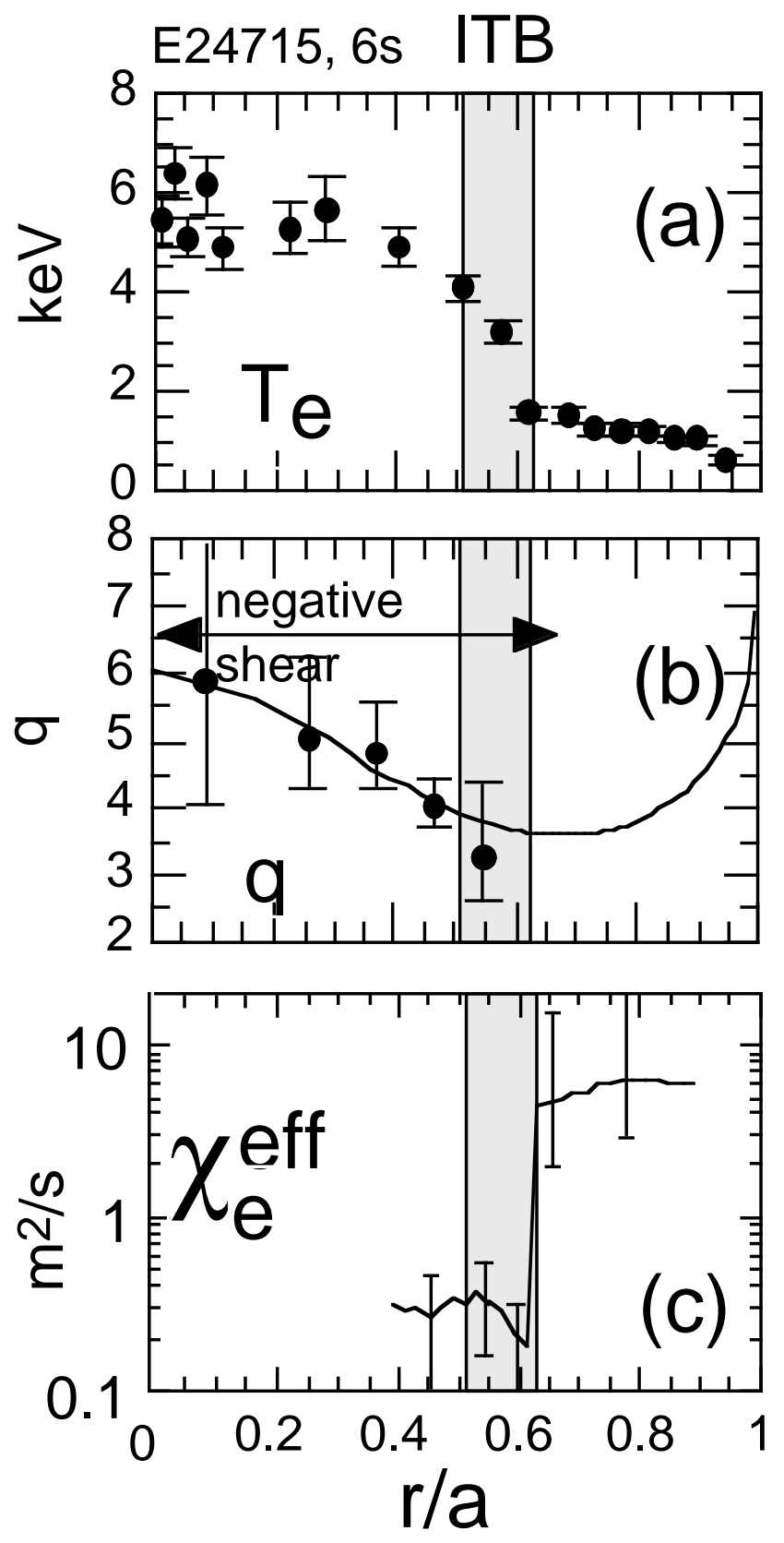

Figure 11. Profiles for a JT-60U plasma for which an electron thermal barrier is inferred from power balance analysis. (a). The electron temperature, showing a pedestal. (b). The q profile. (c). The inferred effective electron thermal conductivity $\chi_{\mathrm{e}}^{\mathrm{ff}}$, evaluated assuming all the power flow is from conduction. From [Fujita, 1997] 

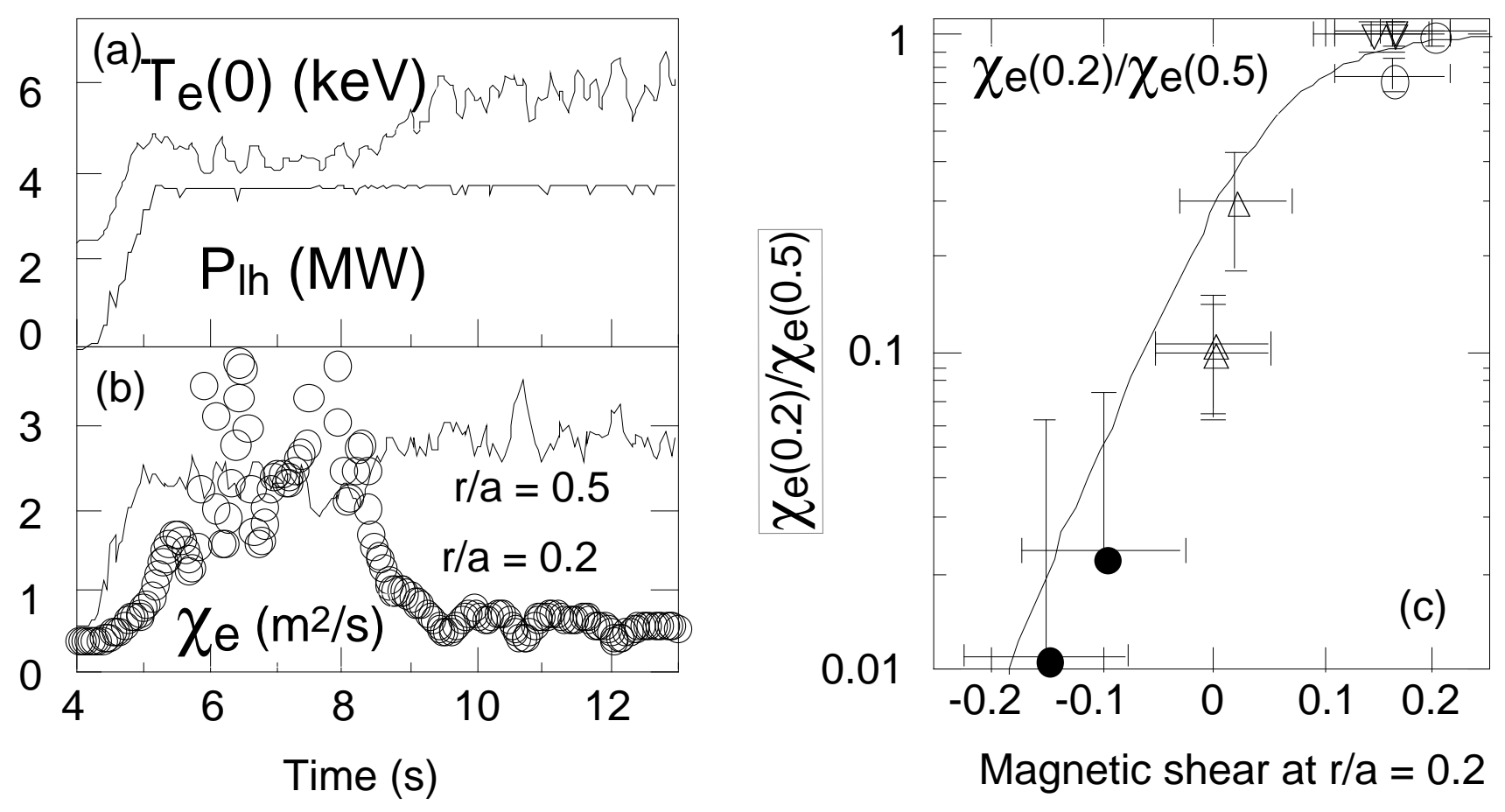

Figure 12. (a). The central electron temperature and LHCD heating power for a Tore Supra discharge with weak magnetic shear. (b). The electron thermal diffusivity $\chi_{\mathrm{e}}$, inferred from power balance, for the same discharge, at two different radii. (c). $\chi_{\mathrm{e}}(0.2) / \chi_{\mathrm{e}}(0.5)$ plotted versus local magnetic shear at $\mathrm{r} / \mathrm{a}=0.2$, highlighting the observed correlation between shear strength and $\chi_{\mathrm{e}}$. From [Litaudon, 1996]. 

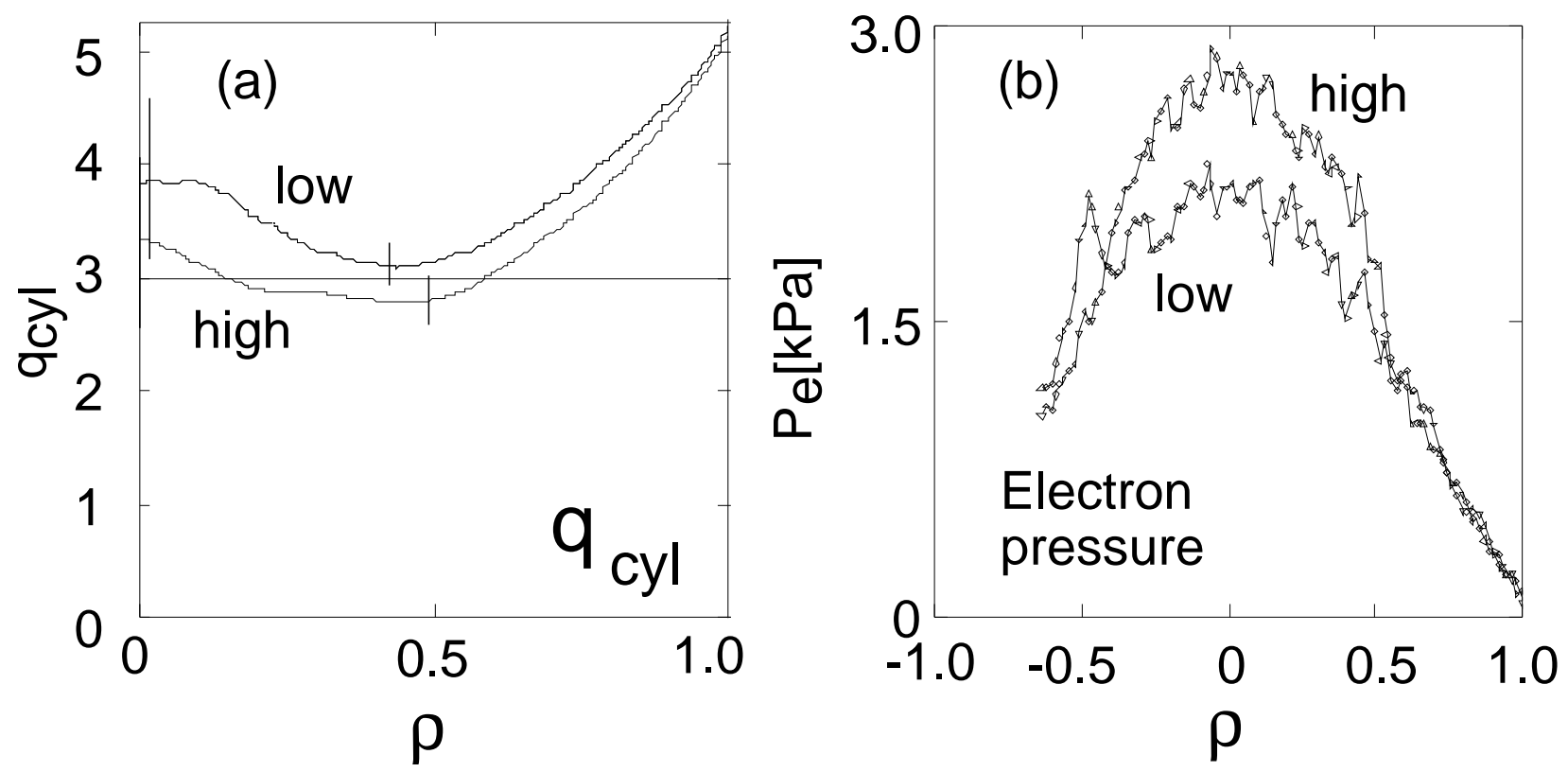

Figure 13. (a) Safety factor of high confinement discharge obtained with ECH heating on RTP . Note that $\mathrm{q}\left(\rho_{\mathrm{dep}}\right)<3$. The $\mathrm{q}$ profile of a discharge with low confinement is shown for comparison. The minimal value of $\mathrm{q}$ in this case is 3.1. (b). Electron pressure profiles for the plasma with lower confinement and with a core transport barrier. Note that both profiles have the same gradients, except for the region near $\rho=0.5$. From [De Baar, 1997]. 


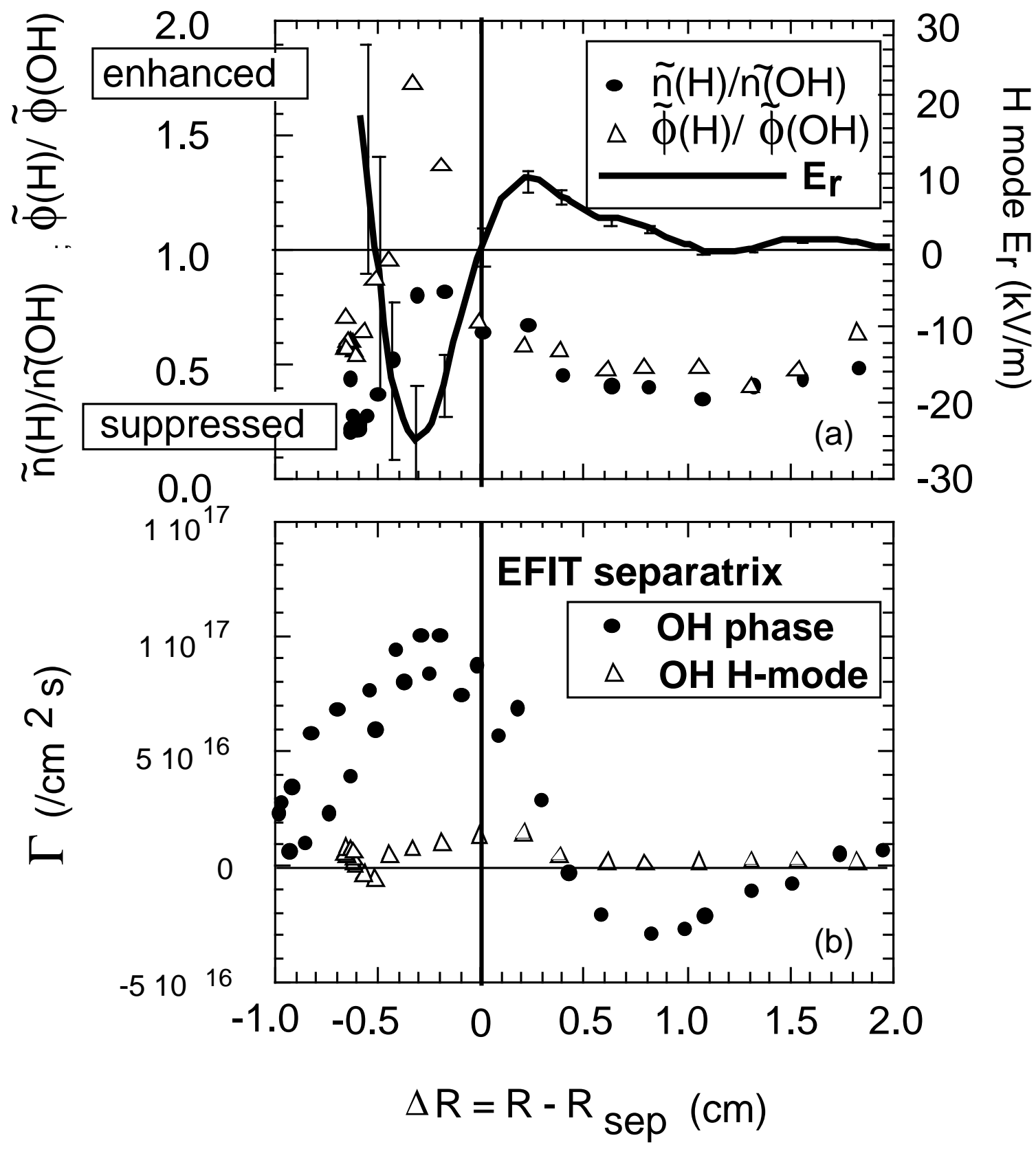

Figure 14. Profiles of $E_{r}$ and the ratios of the absolute RMS density and potential fluctuation amplitudes $\widetilde{\mathrm{n}}$ (circles ) and $\widetilde{\phi}$ (triangles) in the Ohmic H mode to the values in the Ohmic phase. The suppression of $\widetilde{\mathrm{n}}$ is weakest, and $\tilde{\phi}$ is enhanced, where $\mathrm{E}_{\mathrm{r}}^{\prime} \sim 0(\Delta \mathrm{R} \sim=0.3 \mathrm{~cm})$. (b) Profiles of the turbulent particle flux $\Gamma$ in the Ohmic $\mathrm{H}$ mode (triangles) and Ohmic phase (circles). $\Gamma$ is reduced in the $\mathrm{E}_{\mathrm{r}}$ shear layer by up to an order of magnitude in the $\mathrm{H}$ mode, a result of the dephasing of $\widetilde{n}$ and $\widetilde{\phi}$. From [Moyer, 1995]. 
